\title{
New Proofs of the Existence of the Feigenbaum Functions
}

\section{H. Epstein}

Institut des Hautes Etudes Scientifiques, 35, route de Chartres, F-91440 Bures-sur-Yvette, France

\begin{abstract}
A new proof of the existence of analytic, unimodal solutions of the Cvitanović-Feigenbaum functional equation $\lambda g(x)=-g(g(-\lambda x)), \quad g(x)$ $\approx 1-$ const $|x|^{r}$ at 0 , valid for all $\lambda$ in $(0,1)$, is given, and the existence of the Eckmann-Wittwer functions [8] is recovered. The method also provides the existence of solutions for certain given values of $r$, and in particular, for $r=2$, a proof requiring no computer.
\end{abstract}

\section{Notations}

If $z \in \mathbf{C}$, we denote $z^{*}$ its complex conjugate, and reserve the notation $\bar{S}$ to denote the closure of a set $S$.

Let $J$ be an open, possibly empty interval in $\mathbf{R}$. We denote

$$
\mathbf{C}(J)=\{z \in \mathbf{C}: \operatorname{Im} z \neq 0 \text { or } z \in J\} .
$$

In particular, $\mathbf{C}(\emptyset)=\mathbf{C}_{+} \cup \mathbf{C}_{-}$, where

$$
\mathbf{C}_{+}=-\mathbf{C}_{-}=\{z \in \mathbf{C}: \operatorname{Im} z>0\} \text {. }
$$

$\mathbf{F}(J)$ is the real Fréchet space of functions $f$, holomorphic on $\mathbf{C}(J)$, with $f\left(z^{*}\right)^{*}=f(z)$, equipped with the topology of uniform convergence on compact subsets of $\mathbf{C}(J) . \mathbf{P}(J)$ is the subset of $\mathbf{F}(J)$ consisting of the functions $f$ such that $f\left(\mathbf{C}_{+}\right) \subset \overline{\mathbf{C}}_{+}$, and $f\left(\mathbf{C}_{-}\right) \subset \overline{\mathbf{C}}_{-}$. These functions are often called Herglotz or Pick functions.

$\mathbf{P}_{0}(J)$ is the subset of $\mathbf{P}(J)$ consisting of the functions $f$ such that $|f(z) / z| \rightarrow 0$ as $z \rightarrow \infty$ in non-real directions.

\section{Introduction}

The functional equation

$$
g(x)=-\frac{1}{\lambda} g(g(-\lambda x))
$$


was formulated by Cvitanović and Feigenbaum [11] (see also [5]) to explain universal features of period doubling in maps of the interval. This section enumerates a set of constraints for the solutions whose existence is proved in the subsequent sections. These properties are suggested by the accumulated literature.

C1. $g$ is an even $C^{1}$ map of $[-1,1]$ into itself with a unique critical point at 0 , and $g(0)=1$.

C2. There is a real $r>1$, a complex neighborhood of $[0,1]$ in $\mathbf{C}$, and a function $f$, holomorphic in this neighborhood, with $f^{\prime}(0) \neq 0$, such that

$$
g(x)=f\left(x^{r}\right) \text { for } 0 \leqq x \leqq 1 .
$$

In particular, near $0, g(x) \approx 1-$ const $|x|^{r}$. If $g$ satisfies $\mathbf{C} \mathbf{1}$ and $\mathbf{C 2}$, then (1.1) is equivalent to

$$
g(x)=-\frac{1}{\lambda} g(g(\lambda x))
$$

which implies $g(1)=-\lambda$. Since $g$ is decreasing on $(0,1)$, so is $x \rightarrow g(|\lambda| x)-x$, which takes the value 1 at 0 and $g(|\lambda|)-1 \leqq 0$ at 1 . Hence it vanishes at a unique $x_{0} \in(0,1)$, where $g\left(x_{0}\right)=-g\left(x_{0}\right) / \lambda$. Thus if $\lambda \neq-1, g\left(x_{0}\right)=0$, so $g(1) \leqq 0$. It is easily checked that $\lambda=-1$ is incompatible with our hypotheses, as well as $\lambda=0$ or 1 , and so we impose:

C3. $0<\lambda<1$.

Note that this implies $x_{0}>\lambda$ (for otherwise $g\left(x_{0} / \lambda\right) \in[-1,1]$, but $g\left(x_{0} / \lambda\right)$ $=-1 / \lambda<-1)$. Similarly, from $g(g(\lambda))=\lambda^{2}$, it follows $g(\lambda)>\lambda$ since otherwise $g(\lambda) / \lambda \in[-1,1]$, but

$$
g(g(\lambda) / \lambda)=-g\left(\lambda^{2}\right) / \lambda<-g\left(\lambda x_{0}\right) / \lambda=-x_{0} / \lambda<-1 .
$$

For each $r>1$, it appears, from numerical experimentation and existing rigorous results $[11,3,13,1,14,10]$, that a locally unique solution exists, with the above properties, and depends smoothly on $r$, with $\lambda$ an increasing function of $r$.

The study by Eckmann and Wittwer [8] of the asymptotic behavior of the problem as $r \rightarrow \infty$, has shown that, in that limit, $g$ and $f$ degenerate but $f(t)^{r}, x_{0}^{r}, \lambda^{r}$ have non-trivial limits. It is therefore useful to consider:

$$
G(t)=f(t)^{r}=g\left(t^{1 / r}\right)^{r}, \quad y_{0}=x_{0}^{r}, \quad \tau=\lambda^{r} .
$$

Since $f\left(y_{0}\right)=0, G$ is, in general, only analytic on $\left(0, y_{0}\right)$. We also introduce, with [8] and [3],

$$
a(t)=G(\tau t)=g\left(\lambda t^{1 / r}\right)^{r},
$$

which is analytic and decreasing on $\left[0, y_{0} / \tau\right)$.

A straightforward generalization of the facts known from [9] in the case $r=2$ leads to the requirement:

C4. The inverse function of $g \mid(0,1)$ extends to a function $u \in-\mathbf{P}((-1 / \lambda, 1))$. The inverse function on of $f \mid(0,1)$ extends to a function $U \in-\mathbf{P}\left(\left(-\lambda^{-1}, \lambda^{-2}\right)\right)$. 
Clearly this implies, for all $\zeta$ in $\mathbf{C}((-1 / \lambda, 1))$,

$$
U(\zeta)=u(\zeta)^{r}
$$

and that $u$ sends $\mathbf{C}_{-}$into $\{\zeta: 0<\operatorname{Arg} \zeta<\pi / r\}$. Moreover,

$$
U(0)=y_{0}, \quad U(1)=0, \quad U\left(x_{0}\right)=\tau y_{0} .
$$

Taking the inverse function of $a$, and rescaling it by $\frac{1}{y_{0}}$, we define:

$$
\begin{gathered}
V(\zeta)=\frac{1}{y_{0}} a^{-1}\left(y_{0} \zeta\right), \\
\psi(z)=\frac{1}{y_{0}} U(z),
\end{gathered}
$$

and we obtain the conditions:

C5.

$$
\begin{gathered}
V \in-\mathbf{P}\left(\left(0,1 / y_{0} \tau^{2}\right)\right), \quad V(1)=1, \quad V^{\prime}(1)=-\frac{1}{\lambda}, \\
\psi \in-\mathbf{P}\left(\left(-\lambda^{-1}, \lambda^{-2}\right)\right), \quad \psi(0)=1, \quad \psi(1)=0, \\
\psi(z)=V(\psi(-\lambda z)), \\
V(\zeta)=\frac{1}{\tau} \psi\left(\left(y_{0} \zeta\right)^{1 / r}\right) .
\end{gathered}
$$

The conditions C1, C2 define a particular class of solutions of (1.1). There are many others, which are not at all considered in this paper. Some are analytic but have additional critical points in $(0,1)$, and it is likely that they correspond to a bifurcation, in function space, of codimension $>1$. Some are less regular (see e.g. [4]) and may be expected to play a less prominent (or more complicated) role in the dynamics of maps of the interval. Note, in particular, that, inasmuch as the fixed point $g$ is conjectured to attract maps of many one-parameter families, it will attract, in particular, many analytic ones, e.g. $1-\mu|x|^{r}$, whose inverse functions have the properties corresponding to $\mathbf{C 4}$. Since these properties are very stable under limits, $g$ itself can be expected to inherit them.

Several proofs of the existence of solutions satisfying C1-C4 already exist for particular values of $r$ and $\lambda[3,13,1,14,9,8,10]$. Except for [3] and [8], they do little to reveal the branch of special function theory which probably underlies the subject. Nor will this paper shed much light on this, but it is, hopefully, a small step in the right direction. The method of this paper is to look for solutions as fixed points of a map suggested by C5, and to apply the Schauder-Tikhonov theorem [7] by taking advantage of the normality properties of Herglotz functions. Section 3 uses a version $M_{\lambda}$ of this map defined for a fixed value of $\lambda$, and proves the existence of solutions satisfying $\mathbf{C 1}-\mathbf{C 5}$ for every $\lambda \in(0,1)$. Moreover it is possible to reobtain the existence of the Eckmann-Wittwer functions in the limit $\lambda \rightarrow 1$. In fact $M_{\lambda}$ is essentially identical to the map used (and proved to be contractive) in 
[8]; however it is used here in different function spaces. Appendix 2 owes much to [8] and to the ideas of Ecalle reviewed there. It essentially shows that, when suitably reinterpreted, $M_{\lambda}$ has a limit when $\lambda \rightarrow 1$, and gives a direct proof of the existence of the Eckmann-Wittwer functions. But since the Schauder-Tikhonov theorem does not assert any kind of uniqueness, the proof in Sect. 3 implies no continuous dependence of $r$ on $\lambda$, although it is intuitively obvious, and proved in [3] for small $\lambda$, that this dependence is, in fact, analytic; and it is likely that the map $M_{\lambda}$ used in Sect. 3 is, in fact, a contraction. Section 4 describes a version of the method where $r$ is fixed. It is, unfortunately, much less successful, although it does prove the existence of solutions for $r \leqq 14$. In particular, for $r=2$, it provides a proof that requires no other computing machinery than paper and pen. It would be much more interesting to be able to define and solve a fixed point problem for $\psi$ or $V$ regarded as a function of two complex variables, e.g. $z$ and $\lambda$. This remains a possibility for the future. To a certain extent the methods of this paper can be applied to the case of circle maps. This will be described in a paper in preparation by J.-P. Eckmann and myself.

The literature concerning Feigenbaum's theory is very extensive, and only a small part of it appears in the list of references. The reader is referred, in particular, to $[11,12,2,8,16]$ for more detailed scientific as well as bibliographical information.

\section{Classical Results About $\mathbf{P}(J)$}

The properties recalled below can be found e.g. in $[6,15]$.

\subsection{Integral Representation}

Any $f \in \mathbf{P}(J)$ has a unique integral representation:

$$
f(z)=a z+b+\int d \mu(t)\left[\frac{1}{t-z}-\frac{t}{t^{2}+1}\right]
$$

valid for all $z \in \mathbf{C}(J)$. Equivalently, for any $z_{0} \in \mathbf{C}(J)$,

$$
f(z)-f\left(z_{0}\right)=a\left(z-z_{0}\right)+\int d \mu(t)\left[\frac{1}{t-z}-\frac{1}{t-z_{0}}\right] .
$$

Here $\mu$ is a positive measure on $\mathbf{R}$ with support in $\mathbf{R}-J$, such that $\int d \mu(t)(|t|+1)^{-2}<\infty$. For any continuous $\phi$ on $\mathbf{R}$, sufficiently decreasing at $\infty$,

$$
\int \phi(t) d \mu(t)=\lim _{\varepsilon \downarrow 0} \frac{1}{\pi} \int \phi(t) \operatorname{Im} f(t+i \varepsilon) d t .
$$

The constant $a \geqq 0$ is called the angular derivative of $f$ at infinity. Uniformly in any closed angle contained in $\mathbf{C}_{ \pm}$,

$$
\lim _{|z| \rightarrow \infty}|(f(z)-a z) / z|=0 .
$$


We denote $\mathbf{P}_{0}(J)$ the subset of $\mathbf{P}(J)$ consisting of functions for which $a=0$. It is dense in $\mathbf{P}(J)$ : if, e.g., $f$ belongs to $\mathbf{P}(J)$ with $J=(0,1)$ or $(0, \infty)$, then, for $0<s<1$, $f_{s}(z)=f\left(z^{s}\right)$ defines an element $f_{s}$ of $\mathbf{P}_{0}(J)$ which tends to $f$ as $s \rightarrow 1$. This remark can simplify the verification of inequalities such as (3.4), (3.5), etc.

\subsection{Positivity Conditions on Derivatives}

Suppose that $J \neq \emptyset$. Then, for every $z \in J$, and every finite complex sequence $v_{0}, \ldots, v_{N}$,

$$
\sum_{j, k=0}^{N} \frac{f^{(j+k+1)}(z)}{(j+k+1) !} v_{j}^{*} v_{k} \geqq 0 .
$$

In particular $f^{(n)}$ is positive for all odd $n$, and:

$$
S f(z) \equiv \frac{f^{\prime \prime \prime}(z)}{f^{\prime}(z)}-\frac{3}{2}\left[\frac{f^{n}(z)}{f^{\prime}(z)}\right]^{2} \geqq 0 .
$$

\subsection{Special Case of $J=(-\infty, 0)$}

If $f \in \mathbf{P}((-\infty, 0))$, then $f^{(n)}$ is positive for all $n \in \mathbf{N}_{*}$. If, moreover, $f(x) \rightarrow 0$ when $x \rightarrow-\infty$ in $\mathbf{R}$, then $\int(|t|+1)^{-1} d \mu(t)<\infty$, and

$$
f(z)=\int \frac{d \mu(t)}{t-z}
$$

\subsection{Normality}

$\mathbf{P}(\emptyset)$ is a normal family. The same is true of the subset of functions in $\mathbf{P}(J)$ which, on $J$, are bounded in modulus by some fixed $M<\infty$.

\subsection{Iteration of Functions in $\mathbf{P}(\emptyset)$}

Denote $f_{+}$the restriction of $f \in \mathbf{P}(\emptyset)$ to $\mathbf{C}_{+}$. Then (see [15]), either $f_{+}$is an isomorphism of $\mathbf{C}_{+}$, or $f_{+}^{n}$ converges, uniformly on any compact subset of $\mathbf{C}_{+}$, to a constant $C$. There are three possible cases:

1) $C=\infty$ : this can happen only if $a>1$.

2) $C \in \mathbf{R}$.

3) $C \in \mathbf{C}_{+}$: then $C$ is an attractive fixed point of $f_{+}$, i.e. $\left|f_{+}^{\prime}(C)\right|<1$.

\subsection{Final Remark}

Let $f \in \mathbf{P}((b, c))$, not identically 0 , with $-\infty<b<0<c<\infty$ and suppose that $f(0)=0$. Then, on $(b, c), f(x) / x$ is a strictly positive, convex function and, for all $z=x+i y$ such that $b<x<c$,

$$
\frac{|f(z)|}{|z|} \leqq \frac{f(x)}{x}
$$




\section{The Fixed $\lambda$ Method}

For any fixed $\lambda \in(0,1)$, this method sets up a map $M_{\lambda}$ which we first describe informally. Throughout this section, $\lambda$ is fixed in $(0,1)$.

1) Start with a given $\psi_{0} \in-\mathbf{P}\left(\left(-\lambda^{-1}, \lambda^{-2}\right)\right)$, satisfying $\psi_{0}(0)=1, \psi_{0}(1)=0$, and other conditions to be stated later.

2) Define:

$$
V(\zeta)=\frac{1}{\tau} \psi_{0}\left((\zeta / \alpha)^{1 / r}\right),
$$

the real numbers $\tau>0, \alpha>0, r>1$ being adjusted so that

$$
V(1)=1, \quad V^{\prime}(1)=-\frac{1}{\lambda}, \quad \tau=\lambda^{r} .
$$

3) Find $\psi$ such that

$$
\psi(z)=V(\psi(-\lambda z)), \quad \psi(0)=1, \quad \psi(1)=0,
$$

and verify that $\psi$ satisfies all the conditions imposed on $\psi_{0}$. Then define

$$
M_{\lambda} \psi_{0}=\psi \text {. }
$$

To carry out step 3), it will be convenient to introduce auxiliary functions, in particular $W$ :

$$
W(\zeta)=V(V(\zeta)) .
$$

We now study the map $M_{\lambda}$ in detail. Recall that, in this section, $\lambda$ is chosen once and for all in $(0,1)$. In the remainder of this paper, we denote:

$$
A \equiv A(\lambda)=-\frac{1}{\lambda \log \lambda}, \quad B \equiv B(\lambda)=\left(1-\lambda^{2}\right) A(\lambda) .
$$

Note that $A \geqq e$, and $B$ is a decreasing function of $\lambda$ tending to 2 as $\lambda \rightarrow 1$.

\subsection{Determination of $\tau, \alpha$, and $r$}

We start from a fixed $\psi_{0}=1-\hat{\psi}_{0} \in-\mathbf{P}\left(\left(-\lambda^{-1}, \lambda^{-2}\right)\right)$, with $\psi_{0}(0)=1$ and $\psi_{0}(1)=0$. As recalled in Sect. 2, there is a constant $a_{0} \geqq 0$, and a positive measure $\xi_{0}$, with support in

$$
\Sigma(\lambda)=\mathbf{R}-\left(-\lambda^{-1}, \lambda^{-2}\right),
$$

such that, for all $z \in \mathbf{C}\left(\left(-\lambda^{-1}, \lambda^{-2}\right)\right)$,

$$
\frac{\psi_{0}(z)}{1-z}=a_{0}+\int \frac{d \xi_{0}(t)}{(t-z)(t-1)},
$$

and:

$$
0 \leqq \int \frac{d \xi_{0}(t)}{t(t-1)}=1-a_{0}
$$


Rewriting the integrand of (3.2) in the form:

$$
\frac{d \xi_{0}(t)}{t(t-1)} \frac{t}{(t-z)}
$$

and noting that, for $0 \leqq z \leqq \lambda^{-2}$, and $t$ in $\Sigma(\lambda)$,

$$
(1+\lambda z)^{-1} \leqq t(t-z)^{-1} \leqq\left(1-\lambda^{2} z\right)^{-1},
$$

we obtain:

$$
\frac{1}{1+\lambda z} \leqq \frac{\psi_{0}(z)}{1-z} \leqq \frac{1}{1-\lambda^{2} z} \quad\left(0 \leqq z \leqq \lambda^{-2}\right)
$$

Similarly, from

$$
-\psi_{0}^{\prime}(z)=a_{0}+\int \frac{d \xi_{0}(t)}{(t-z)(t-1)} \frac{(t-1)}{(t-z)},
$$

it follows that, for $-\lambda^{-1} \leqq z \leqq 1$,

$$
\frac{\left(1-\lambda^{2}\right)}{\left(1-\lambda^{2} z\right)(1-z)} \leqq \frac{-\psi_{0}^{\prime}(z)}{\psi_{0}(z)} \leqq \frac{(1+\lambda)}{(1+\lambda z)(1-z)},
$$

and from

$$
-\psi_{0}^{\prime \prime}(z)=2 \int \frac{d \xi_{0}(t)}{(t-z)^{2}} \frac{1}{t-z},
$$

it follows that, for $-\lambda^{-1} \leqq z \leqq \lambda^{-2}$,

$$
-\frac{2 \lambda}{1+\lambda z} \leqq \frac{\psi_{0}^{\prime \prime}(z)}{\psi_{0}^{\prime}(z)} \leqq \frac{2 \lambda^{2}}{1-\lambda^{2} z} .
$$

Suppose now that, for some positive $\tau, \alpha$, and $r>1$, the function

$$
V(\zeta)=\frac{1}{\tau} \psi_{0}\left((\zeta / \alpha)^{1 / r}\right)
$$

is defined and differentiable at $\zeta=1$, and satisfies $V(1)=1, V^{\prime}(1)=-\lambda^{-1}$. Then we must have:

$$
\psi_{0}\left(z_{1}\right)=\tau, \quad z_{1}=\alpha^{-1 / r}
$$

and:

$$
\frac{z_{1} \psi_{0}^{\prime}\left(z_{1}\right)}{\psi_{0}\left(z_{1}\right)}=-\frac{r}{\lambda} .
$$

If we also require $\tau=\lambda^{r}$, i.e. $r=\log \tau / \log \lambda$, we must have:

$$
\begin{aligned}
q\left(z_{1}\right) & =0, \\
q(z) & \equiv \frac{\psi_{0}^{\prime}(z)}{\psi_{0}(z)}-\frac{A}{z} \log \psi_{0}(z) .
\end{aligned}
$$


The function $q$ is smooth on $[0,1)$, with $q(0)=\psi_{0}^{\prime}(0)(1-A)>0$. When $z \rightarrow 1$, it behaves like $-(1-z)^{-1}-A \log (1-z)$, and tends to $-\infty$. It therefore has zeroes in $(0,1)$, and we take $z_{1}$ as any one of them. Actually it will shortly be seen that there is only one such zero.

Having chosen $z_{1}$ in this way, we define:

$$
\tau=\psi_{0}\left(z_{1}\right), \quad r=\frac{\log \tau}{\log \lambda}, \quad \alpha=z_{1}^{-r} .
$$

Since $\psi_{0}$ is strictly decreasing on $\left[-\lambda^{-1}, \lambda^{-2}\right]$, we have: $0<\tau<1, r>0, \alpha>1$.

\subsection{Lower Bounds on $z_{1}$ and $1 / \tau$}

Since $\psi_{0}$ is negative on $\left(1, \lambda^{-2}\right)$, the function $\log \psi_{0}$ belongs to $-\mathbf{P}\left(\left(-\lambda^{-1}, 1\right)\right)$. When $z \in \mathbf{C}_{-}$, clearly $0<\operatorname{Im} \log \psi_{0}(z)<\pi$, so that the angular derivative of this function at infinity vanishes. It has, therefore, an integral representation, for $z \in \mathbf{C}\left(\left(-\lambda^{-1}, 1\right)\right)$

$$
\log \psi_{0}(z)=-\int \sigma(t) d t\left[\frac{1}{t-z}-\frac{1}{t}\right]
$$

where $\sigma \in L^{\infty}$ has support in $\mathbf{R}-\left(-\lambda^{-1}, 1\right)$, and $0 \leqq \sigma \leqq 1$. Moreover, for $t \in\left(1, \lambda^{-2}\right), \log \psi_{0}(t+i 0)=\log \left[-\psi_{0}(t)\right]-i \pi$, so that $\sigma(t)=1$ there. The function $q$ has the integral representation, in $\mathbf{C}\left(\left(-\lambda^{-1}, 1\right)\right)$,

$$
q(z)=\int \frac{\sigma(t) d t}{t-z}\left[\frac{A}{t}-\frac{1}{t-z}\right] \text {. }
$$

Let $0<z<1$. For $t \leqq-\lambda^{-1}$, the integrand is positive. For $t \geqq 1$, it has the sign of $(A-1) t-A z \geqq(A-1)-A z$, and is certainly $>0$ if $z<1-A^{-1}$. We conclude:

$$
z_{1}>1-\lambda \log \frac{1}{\lambda}>\lambda
$$

The last inequality follows from the usual inequality $\log x \leqq x-1$ for all $x>0$, strict for $x \neq 1$. Since $r>0$, it follows that:

$$
\alpha<\frac{1}{\tau}
$$

To get stronger bounds on $z_{1}$, we separate, in the integral in (3.13), the contributions from $\left[1, \lambda^{-2}\right]$ and from the rest of the support of $\sigma$. For $0<z<1$, as already noted, the contribution from $\left(-\infty,-\lambda^{-1}\right)$ is positive. For $t \geqq \lambda^{-2}$, the integrand has the sign of $(A-1) t-A z$, which is minorized by:

$$
(A-1) \lambda^{-2}-A=A \lambda^{-2}\left(1-\lambda \log \frac{1}{\lambda}-\lambda^{2}\right)>0 \text {. }
$$

Therefore, for $0<z<1, q(z) \geqq q_{2}(z)$, where:

$$
q_{2}(z)=\int_{1}^{1 / \lambda^{2}} d t\left[\frac{A}{z}\left(\frac{1}{t-z}-\frac{1}{t}\right)-\frac{1}{(t-z)^{2}}\right]=\frac{A}{z} \log \left[\frac{1-\lambda^{2} z}{1-z}\right]-\frac{1-\lambda^{2}}{(1-z)\left(1-\lambda^{2} z\right)} .
$$


It is convenient to use the variable

$$
\xi=\frac{1-\lambda^{2} z}{1-z}, \quad z=\frac{\xi-1}{\xi-\lambda^{2}} .
$$

This gives:

$$
\begin{gathered}
\left(1-\lambda^{2}\right) z q_{2}(z)=\chi(\xi)-\xi, \\
\chi(\xi)=B \log \xi+1+\lambda^{2}-\frac{\lambda^{2}}{\xi} .
\end{gathered}
$$

The function $\chi$ is increasing and concave on $(0, \infty)$, and $\chi(\xi)-\xi$ vanishes at $\xi=1$ and at a unique $\xi>1$. Since $1 \leqq \xi \leqq \xi$ is equivalent to $\chi(\xi) \geqq \xi$, it follows that $\xi$ is a lower bound for $\xi_{1}=\left(1-\lambda^{2} z_{1}\right) /\left(1-z_{1}\right)$. Applying the bounds (3.4) shows that:

$$
\frac{1}{\tau} \geqq \xi_{1} \geqq \xi .
$$

It is immediate to verify that:

$$
\chi\left(\frac{1}{\lambda}\right)-\frac{1}{\lambda}=1-\lambda+\lambda^{2}-\lambda^{3}>0,
$$

from which it follows that $\tau<\lambda$ and hence $r>1$. But we need the more precise bound:

$$
\xi>\lambda^{-(1+y)}, \quad y=\frac{2 \lambda^{2}}{1-\lambda^{2}} .
$$

Inserting $\xi=\lambda^{-(1+y)}$ into $\chi(\xi)-\xi$ gives:

$$
\frac{1}{\lambda}\left[1+\lambda+\lambda^{2}-\exp \left(\frac{2 \lambda^{2}}{1-\lambda^{2}} \log \frac{1}{\lambda}\right)\right]+\lambda^{2}\left(1-\lambda^{1+y}\right) .
$$

The positivity of the first bracket follows from the

Lemma 1. For $0 \leqq x \leqq 1$, the quantity

$$
\left(1-x^{2}\right) \log \left(1+x+x^{2}\right)+2 x^{2} \log x
$$

is non-negative, and vanishes only at 0 and 1 .

The straightforward and tedious proof of this is sketched in Appendix 1.

As a consequence of (3.21),

$$
\frac{1}{\tau}>\left(\frac{1}{\lambda}\right)^{1+y}, \quad r>1+\frac{2 \lambda^{2}}{1-\lambda^{2}} .
$$

We also note:

$$
\frac{1}{\tau}>3
$$

Indeed, $\chi(3)-3>1+B-3>0$. 


\subsection{Uniqueness of $z_{1}$}

The derivative of $z q(z)$ in $(0,1)$ satisfies:

$$
\frac{\psi_{0}(z)}{\psi_{0}^{\prime}(z)} \frac{d}{d z}(z q(z))=-z \frac{\psi_{0}^{\prime}(z)}{\psi_{0}(z)}-A+1+z \frac{\psi_{0}^{\prime \prime}(z)}{\psi_{0}^{\prime}(z)} \geqq-z \frac{\psi_{0}^{\prime}(z)}{\psi_{0}(z)}-A+\frac{1-\lambda z}{1+\lambda z}
$$

[by (3.6)]. At $z=z_{1}$, this gives:

$$
\left|(z q(z))^{\prime}\right|_{z=z_{1}} \geqq A \log \frac{1}{\tau}\left[A\left(\log \frac{1}{\tau}-1\right)+\frac{1-\lambda}{1+\lambda}\right] .
$$

Thus, the derivative of $z q(z)$ at every zero it has in $(0,1)$ is strictly negative. Therefore $z q(z)$ has only one zero in $(0,1)$.

\subsection{Lower Bound on $\tau$}

It is clear that $\tau>0$, since $z_{1} \neq 1$. In this subsection, we prove the existence of a (strictly positive) lower bound for $\tau$, which depends only on $\lambda$. In Subsect. 3.11, using additional constraints on $\psi_{0}$, we shall prove the existence of a lower bound uniform in $\lambda$ as $\lambda \rightarrow 1$.

Separating, in the integral representation of $\log \psi_{0}$, the contribution of $\left[1, \lambda^{-2}\right]$ from the rest gives:

$$
\log \psi_{0}(z)+\log \frac{1-\lambda^{2} z}{1-z}=-\int_{\Sigma} \sigma(t) d t \frac{z}{t(t-z)} .
$$

[Recall that $\Sigma=\mathbf{R}-\left(-\lambda^{-1}, \lambda^{-2}\right)$.] Letting $z$ tend to 1 from below gives:

$$
k \equiv \int_{\Sigma} \frac{\sigma(t) d t}{t(t-1)}=\log \left[\frac{-1}{\psi_{0}^{\prime}(1)\left(1-\lambda^{2}\right)}\right] .
$$

Letting $z$ tend to 1 in the inequalities (3.4) we find:

$$
\frac{1}{1+\lambda} \leqq-\psi_{0}^{\prime}(1) \leqq \frac{1}{1-\lambda^{2}}
$$

and hence:

$$
k \leqq \log \frac{1}{1-\lambda} \leqq \frac{\lambda}{1-\lambda}
$$

The function $q_{1}(z)=q(z)-q_{2}(z)$ has been shown to be positive in $(0,1)$. To majorize it, we write it in the form:

$$
q_{1}(z)=\int_{\Sigma} \frac{\sigma(t) d t}{t(t-1)} I(t, z), \quad I(t, z)=\frac{(A-1) t-A z}{(t-z)^{2}}(t-1) .
$$

It is easy to see that, when $t \in \Sigma$ and $z \in(0,1)$,

$$
I(t, z) \leqq(A-1)(1+\lambda),
$$


and so, for $z \in(0,1)$,

$$
\begin{gathered}
q_{1}(z) \leqq(A-1)(1+\lambda) k \\
\left(1-\lambda^{2}\right) z q(z) \leqq\left(1-\lambda^{2}\right) z q_{2}(z)+\left(1-\lambda^{2}\right)(A-1)(1+\lambda) k .
\end{gathered}
$$

Assume that

$$
\left(1-\lambda^{2}\right)(A-1)(1+\lambda) k+1+\lambda^{2} \leqq K .
$$

Then, using again the variable $\xi$ defined in (3.17), we get:

$$
\chi(\xi)-\xi \leqq\left(1-\lambda^{2}\right) z q(z) \leqq \chi(\xi)-\xi-1-\lambda^{2}+K,
$$

with $\chi$ as in (3.19). In particular,

$$
\left(1-\lambda^{2}\right) z q(z) \leqq K+B \log \xi-\xi \equiv S(\xi) .
$$

To obtain an upper bound for the root of the right-hand side and hence for $\xi_{1}$, we may e.g. note that $S^{\prime}(\xi)=B / \xi-1$, so that, for $\xi>2 B$,

$$
S(\xi)<S(2 B)-\frac{1}{2}(\xi-2 B) .
$$

This becomes negative if $\xi>2 S(2 B)+2 B$, and a fortiori if $\xi>2(K+B \log B)$, and this is then an upper bound for $\xi_{1}$. The bounds (3.4) give:

$$
\frac{1}{\tau} \leqq \frac{1+\lambda z_{1}}{1-z_{1}}=\frac{\xi_{1}-\lambda}{1-\lambda}
$$

Remark. Inserting (3.27) into the left-hand side of (3.28) leads to:

$$
S(\xi) \leqq \frac{(1+\lambda)^{2}}{\log 1 / \lambda}+1+B \log \xi-\xi .
$$

For $\lambda \leqq e^{-2}$, this is negative when $\xi$ is given the value

$$
\xi=\lambda^{-(1+Y)}, \quad Y=\frac{4 \lambda}{\log 1 / \lambda} .
$$

By (3.30), this implies:

$$
r<1+\frac{1}{\log 1 / \lambda}\left[4 \lambda-\log \left(1-e^{-2}\right)\right]
$$

which confirms that $r \rightarrow 1$ as $\lambda \rightarrow 0$.

\subsection{Definition of the Functions $V$ and $W$}

We can now define:

$$
V(\zeta)=\frac{1}{\tau} \psi_{0}\left((\zeta / \alpha)^{1 / r}\right)
$$

Since $r>1,-V$ is a Herglotz function. It is defined, real and analytic at the real points in $\left(0, \alpha \tau^{-2}\right)$. In particular:

$$
V(1)=1, \quad V^{\prime}(1)=-\frac{1}{\lambda}, \quad V(\alpha)=0 .
$$


This function satisfies the following identities, where we denote $z=(\zeta / \alpha)^{1 / r}$ :

$$
\begin{gathered}
V^{\prime}(\zeta)=\frac{1}{\tau r} \frac{z}{\zeta} \psi_{0}^{\prime}(z), \\
\frac{V^{\prime \prime}(\zeta)}{V^{\prime}(\zeta)}=-\frac{1}{\zeta r}\left[r-1-z \frac{\psi_{0}^{\prime \prime}(z)}{\psi_{0}^{\prime}(z)}\right], \\
S V(\zeta)=S \psi_{0}(z)\left[\frac{z}{r \zeta}\right]^{2}+\frac{1}{2 \zeta^{2}}\left(1-r^{-2}\right) .
\end{gathered}
$$

(Recall that $S \psi_{0}$ and $S V$ denote the Schwarzian derivatives of $\psi_{0}$ and $V$, respectively: see 2.2.) From (3.34) and the inequalities (3.6), it follows, when $\zeta \in\left(0, \alpha \tau^{-2}\right)$,

$$
r-1-\frac{2 \lambda^{2} z}{1-\lambda^{2} z} \leqq-r \zeta \frac{V^{\prime \prime}(\zeta)}{V^{\prime}(\zeta)} \leqq r-1+\frac{2 \lambda z}{1+\lambda z} .
$$

In particular when $0<\zeta \leqq \alpha$, i.e. $0<z \leqq 1$, using the first inequality in (3.36), and the lower bound on $r$ given by (3.22), we see that $V$ is convex. Since $V^{\prime}(1)=-1 / \lambda$, this implies $V(\zeta) \geqq 1-(\zeta-1) / \lambda$ in $(0, \alpha)$, and so:

$$
\alpha>1+\lambda .
$$

We also define

$$
W(\zeta)=V(V(\zeta))
$$

and it will be convenient to introduce:

$$
\hat{V}(\zeta)=1-V(1-\zeta), \quad \hat{W}(\zeta)=1-W(1-\zeta)=\hat{V}(\hat{V}(\zeta))
$$

Both $W$ and $\hat{W}$ are Herglotz functions. On the reals, $W$ is defined, real and holomorphic on $(0, \alpha): W(\alpha)=V(0)=1 / \tau$, and $W(0)=V(1 / \tau)$ is defined since $1 / \tau<\alpha / \tau^{2}$.

It is clear that $W(0)<0$, i.e. $\hat{W}(1)>1$, because we have shown that $1 / \tau>\alpha$, but we need more precise bounds.

\subsection{Lower Bounds on $\hat{W}(1)$}

We start by applying the inequalities (3.36) to the case $\zeta=1$, and we find:

$$
0<-\frac{V^{\prime \prime}(1)}{V^{\prime}(1)}<1
$$

Further, by (3.35) and the positivity of $S \psi_{0}$,

$$
S V(\zeta) \geqq \frac{1-r^{-2}}{2 \zeta^{2}}, \quad 0<\zeta<\alpha \tau^{-2} .
$$

Since $W=V \circ V$,

$$
\begin{gathered}
W^{\prime}(\zeta)=V^{\prime}(V(\zeta)) V^{\prime}(\zeta), \\
\frac{W^{\prime \prime}(\zeta)}{W^{\prime}(\zeta)}=\frac{V^{\prime \prime}(V(\zeta))}{V^{\prime}(V(\zeta))} V^{\prime}(\zeta)+\frac{V^{\prime \prime}(\zeta)}{V^{\prime}(\zeta)} \\
S W(\zeta)=S V(V(\zeta)) V^{\prime}(\zeta)^{2}+S V(\zeta)
\end{gathered}
$$


When $\zeta=1$, combining (3.40) and (3.43) gives:

$$
0 \leqq \frac{W^{\prime \prime}(1)}{W^{\prime}(1)}=-\frac{\hat{W}^{\prime \prime}(0)}{\hat{W}^{\prime}(0)} \leqq \frac{1}{\lambda}-1 .
$$

We now apply (3.44) and (3.41) to get:

$$
S W(\zeta) \geqq \frac{V^{\prime}(\zeta)^{2}\left(1-r^{-2}\right)}{2 V(\zeta)^{2}}+\frac{\left(1-r^{-2}\right)}{2 \zeta^{2}} .
$$

For $0<\zeta<1$, the convexity of $V$ implies:

$$
-V^{\prime}(\zeta) \geqq \frac{V(\zeta)-1}{1-\zeta},
$$

hence

$$
-\frac{V^{\prime}(\zeta)}{V(\zeta)} \geqq \frac{1}{1-\zeta-\frac{1}{V^{\prime}(\zeta)}} \geqq \frac{1}{1-\zeta+\lambda}
$$

It follows that

$$
2 S W(\zeta) \geqq\left(1-r^{-2}\right)\left[\frac{1}{(1-\zeta+\lambda)^{2}}+\frac{1}{\zeta^{2}}\right]
$$

and hence

$$
2 S \hat{W}(\zeta) \geqq\left(1-r^{-2}\right)\left[\frac{1}{(\zeta+\lambda)^{2}}+\frac{1}{(1-\zeta)^{2}}\right] .
$$

In $(0,1)$, the right-hand side has a minimum at $\zeta=(1-\lambda) / 2$, and, using the lower bound on $r$ in (3.22), we get

$$
S \hat{W}(\zeta) \geqq s(\lambda) \equiv \frac{16 \lambda^{2}}{\left(1+\lambda^{2}\right)^{2}(1+\lambda)^{2}} .
$$

It follows that, for $0<\zeta<1$,

$$
\frac{d}{d \zeta}\left[\frac{\hat{W}^{\prime \prime}(\zeta)}{\hat{W}^{\prime}(\zeta)}\right]=S \hat{W}(\zeta)+\frac{1}{2}\left[\frac{\hat{W}^{\prime \prime}(\zeta)}{\hat{W}^{\prime}(\zeta)}\right]^{2} \geqq s(\lambda),
$$

and so, using (3.45),

$$
\begin{gathered}
\frac{\hat{W}^{\prime \prime}(\zeta)}{\hat{W}^{\prime}(\zeta)} \geqq-\left(\frac{1}{\lambda}-1\right)+s \zeta, \\
\log \hat{W}^{\prime}(\zeta) \geqq \log \lambda^{-2}-\left(\frac{1}{\lambda}-1\right) \zeta+s \zeta^{2} / 2 .
\end{gathered}
$$

The function $\lambda \rightarrow-2 \log \lambda-\lambda^{-1}+1$ has a unique maximum at $1 / 2$ in $(0,1)$, vanishes at 1 , and takes the value $3-e$ at $\lambda=1 / e$. It is thus positive for $\lambda \geqq 1 / e$, and we conclude that for $\lambda \geqq 1 / e$ and $0<\zeta<1$,

$$
\hat{W}^{\prime}(\zeta) \geqq \exp \left(s \zeta^{2} / 2\right) \geqq 1+s \zeta^{2} / 2, \quad \hat{W}(\zeta) \geqq \zeta\left(1+\frac{s \zeta^{2}}{6}\right) .
$$


We now remark that, since $-V(\zeta)$ and $W(\zeta)$ have been defined as Herglotz functions of $\zeta^{1 / r}$, with $r>1$, they have zero angular derivative at infinity. In particular, for $\zeta \in \mathbf{C}((1-\alpha, 1))$,

$$
\frac{\hat{W}(\zeta)}{\zeta}=\int \frac{d u(t)}{t(t-\zeta)}=\int \frac{d u(t)}{t^{2}} \frac{t}{t-\zeta},
$$

where $u$ is a positive measure with support in $\mathbf{R}-(1-\alpha, 1)$, and $\int d u(t) t^{-2}=\lambda^{-2}$. (Recall that $1-\alpha<-\lambda$.)

Therefore, for $\zeta \in[0,1)$,

$$
\hat{W}(\zeta) \geqq \frac{\zeta}{\lambda(\lambda+\zeta)} \geqq \zeta+\zeta\left[\frac{1}{\lambda(\lambda+1)}-1\right] .
$$

(In fact this bound would hold even if the angular derivative of $\hat{W}$ at infinity did not vanish.) The last bracket in (3.50) is positive for $\lambda<(\sqrt{5}-1) / 2 \sim 0.61$ and, in particular for $0<\lambda \leqq 1 / 2$,

$$
\hat{W}(\zeta) \geqq \zeta\left(1+\frac{\zeta^{2}}{3}\right) .
$$

On the other hand,

$$
4[s(\lambda)]^{-1 / 2}=\frac{1}{\lambda}+1+\lambda+\lambda^{2}
$$

is a convex function of $\lambda$ in $(0,1)$ and so for $\lambda \in[1 / 2,1]$,

$$
4[s(\lambda)]^{-1 / 2} \leqq \max \left(4, \frac{15}{4}\right)=4,
$$

so that $s(\lambda) \geqq 1$, and, by $(3.48), \hat{W}(\zeta)>\zeta\left(1+\zeta^{2} / 6\right)$. Thus:

Lemma 2. There exists a number $a \geqq \frac{1}{6}$ such that, for all $\lambda \in(0,1), \zeta \in[0,1]$,

$$
\hat{W}(\zeta) \geqq \zeta\left(1+a \zeta^{2}\right)
$$

3.7. Upper Bound on $\hat{W}(1)$

Recall that

$$
W(0)=V\left(\frac{1}{\tau}\right)=\frac{1}{\tau} \psi_{0}\left(z_{2}\right)
$$

where

$$
1<z_{2}=(\tau \alpha)^{-1 / r}=z_{1} \lambda^{-1}<\lambda^{-2} .
$$

Using the bound (3.4) on $\psi_{0}$, we find:

$$
-\psi_{0}\left(z_{2}\right) \leqq \frac{1}{\lambda} \frac{z_{1}-\lambda}{1-\lambda z_{1}} \leqq \frac{1}{\lambda}
$$

and so:

$$
W(0) \geqq-\frac{1}{\tau \lambda}, \quad \hat{W}(1) \leqq 1+\frac{1}{\tau \lambda}=1+\left(\frac{1}{\lambda}\right)^{r+1}
$$



Recall that we have proved the existence of an upper bound for $\frac{1}{\tau}$ depending only
on $\lambda$.

We also note that $\hat{W}(1-\alpha)=1-1 / \tau$, and since $1-\alpha<-\lambda, \hat{W}(1-\alpha) /(1-\alpha)$ $\leqq 1 / \tau \lambda$. Together with (3.52) and the remarks in Subsect. 2.6, this shows that for all $\zeta$ with $1-\alpha \leqq \operatorname{Re} \zeta \leqq 1$,

$$
\frac{|\hat{W}(\zeta)|}{|\zeta|} \leqq 1+\frac{1}{\tau \lambda}
$$

\subsection{Definition of $\psi$}

Our purpose is now to construct a function $\psi=1-\hat{\psi}$ in $-\mathbf{P}\left(\left(-\lambda^{-1}, \lambda^{-2}\right)\right)$, satisfying:

$$
\begin{aligned}
& \psi(z)=V(\psi(-\lambda z))=W\left(\psi\left(\lambda^{2} z\right)\right), \\
& \psi(0)=1, \quad \psi(1)=0,
\end{aligned}
$$

or, equivalently,

$$
\begin{aligned}
& \hat{\psi}(z)=\hat{V}(\hat{\psi}(-\lambda z))=\hat{W}\left(\hat{\psi}\left(\lambda^{2} z\right)\right), \\
& \hat{\psi}(0)=0, \quad \hat{\psi}(1)=1 .
\end{aligned}
$$

Recall that:

$$
\begin{aligned}
& \hat{V} \in-\mathbf{P}\left(\left(1-\alpha \tau^{-2}, 1\right)\right), \quad \hat{V}(0)=0, \quad \hat{V}^{\prime}(0)=-\lambda^{-1}, \\
& \hat{W}=\hat{V} \circ \hat{V} \in \mathbf{P}((1-\alpha, 1)), \quad \hat{W}(0)=0, \quad \hat{W}^{\prime}(0)=\lambda^{-2} .
\end{aligned}
$$

As it is well-known, because $\lambda<1$, it is always possible to construct a unique function $\Psi$, holomorphic in a small disk around 0 , and satisfying there:

$$
\Psi(z)=\hat{V}(\Psi(-\lambda z))=\hat{W}\left(\Psi\left(\lambda^{2} z\right)\right), \quad \Psi(0)=0, \quad \Psi^{\prime}(0)=1 .
$$

For the sake of definiteness, we state the following lemma, which it is straightforward to verify (and by no means the best possible estimate):

Lemma 3. Let $f$ be a function holomorphic in $\{z \in \mathbf{C}:|z|<T\}$ and satisfying there, for some $M>0, \omega \in \mathbf{C}, 0<|\omega|=s<1$,

$$
\left|f^{\prime}(z)-\frac{1}{\omega}\right| \leqq 2 M|z|, \quad\left|f(z)-\frac{z}{\omega}\right| \leqq M\left|z^{2}\right| .
$$

Let $0<s<\kappa<1$ and $0<s R<\min \left[T / 2,(\kappa-s) / 4 M s^{2}\right]$. Then the mapping

$$
K_{f} h(z)=f(h(\omega z))
$$

is well defined on the class of the functions $h$ which are holomorphic on $\{z \in \mathbf{C}:|z|<R\}$ and satisfy there

$$
|h(z)-z|<\left|z^{2}\right| / s R \text {. }
$$

It sends this class into itself and is a contraction with ratio $\kappa$ in the distance

$$
\left\|h_{1}-h_{2}\right\|=\sup \left\{\left|z^{-2}\left[h_{1}(z)-h_{2}(z)\right]\right|:|z|<R\right\} .
$$


To apply this lemma, we derive from (3.49), first for real $\zeta$, by the usual estimates, then for complex $\zeta$ by the remark in Subsect. 2.6, that, for $|\zeta|<3 \lambda / 4$, $|\hat{W}(\zeta) / \zeta|<4 \lambda^{-3}$, hence by the Cauchy inequalities,

$$
\left|\hat{W}^{\prime \prime}(\zeta)\right|<24 \lambda^{-4} \text { for }|\zeta|<\lambda / 2 \text {. }
$$

We can take, in the lemma, $f=\hat{W}, T=\lambda / 2, M=12 \lambda^{-4}, s=\lambda^{2}, \kappa=\left(1+\lambda^{2}\right) / 2$, and $R=\min \left\{\left(1-\lambda^{2}\right) / 96 \lambda^{2}, 1 / 4 \lambda\right\}$.

In the disk $\{z:|z|<R\}, \Psi$ is the limit of a uniformly convergent sequence:

$$
\Psi_{n}(z)=\hat{W}^{n}\left(\lambda^{2 n} z\right)
$$

which satisfies $\left|\Psi_{n}(z)\right|<T$ for $|z|<R$.

We can now proceed to extend $\Psi$ outside of the small disk by using the functional equation in (3.56). Note that, inside the disk, $\operatorname{Im} z>0$ implies $\operatorname{Im} \Psi(z) \geqq 0$, the equality being possible only if $\Psi$ is a constant: but this is excluded by $\Psi^{\prime}(0)=1$. Hence, in $\mathbf{C}_{+} \cup \mathbf{C}_{-}$, there never is any obstruction to extending $\Psi$, which is thus a Herglotz function (this also follows from Vitali's theorem). On $R_{+}$, we claim that $\Psi$ continuously extends to a segment $\left[0, \gamma \lambda^{-2}\right]$ on which it assumes all the values in $[0, \hat{W}(1)]$. Indeed $\Psi$ is clearly increasing as far as it can be extended. If its value never reaches $\hat{W}(1)$, then it can never reach 1 . But then $\hat{W}(\Psi(z))$ is always defined, so $\Psi$ extends to all of $\mathbf{R}_{+}$. It also extends to all of $\mathbf{R}_{-}$by $\Psi(z)$ $=\hat{V}(\Psi(-\lambda z))$. This implies that $\Psi(z) \equiv z$, absurd since $\Psi(z)<1$ by assumption for $z>0$. Therefore $\Psi\left(\mathbf{R}_{+}\right)$contains $(0, \hat{W}(1))$. In particular there is a $\gamma>0$ such that $\Psi(\gamma)=1$, and hence $\Psi\left(\gamma \lambda^{-2}\right)=\hat{W}(1)$, and $\Psi\left(-\gamma \lambda^{-1}\right)=\hat{V}(1)=1-1 / \tau$.

We can now define $\hat{\psi}(z)=\Psi(\gamma z)$, which satisfies the requirements in (3.55), and $\psi=1-\hat{\psi}$. Note that these functions are continuous at the ends of their real interval of analyticity, $\left(-\lambda^{-1}, \lambda^{-2}\right)$, with:

$$
\begin{gathered}
\hat{\psi}\left(-\lambda^{-1}\right)=1-1 / \tau, \quad \psi\left(-\lambda^{-1}\right)=1 / \tau, \\
\hat{\psi}\left(\lambda^{-2}\right)=\hat{W}(1), \quad \psi\left(\lambda^{-2}\right)=W(0) .
\end{gathered}
$$

The map $M_{\lambda}$ is defined by $M_{\lambda} \psi_{0}=\psi$.

Note that (3.57) and (3.52) imply that $|\psi(z)| \leqq 1 / \tau \lambda$ for all $z \in\left(-\lambda^{-1}, \lambda^{-2}\right)$, and hence there is a constant $C_{1}(\lambda)>0$, depending only on $\lambda$, such that $|\psi(z)| \leqq C_{1}(\lambda)$ in $\left(-\lambda^{-1}, \lambda^{-2}\right)$.

\subsection{Continuity of the Map $M_{\lambda}$}

Recall that $z_{1}$ was defined as the unique zero of the function $q$ in $(0,1)$. The bounds obtained in the preceding sections, in particular those of Subsect. 3.3, make it clear that $z_{1}$ is a continuous function of $\psi_{0}$; recall that we are using the topology of the Fréchet space $\mathbf{F}\left(\left(-\lambda^{-1}, \lambda^{-2}\right)\right)$. [For example $z_{1}$ is the integral of $(2 \pi i)^{-1} t f^{\prime}(t) f(t)^{-1} d t$ on a small contour surrounding it, with $f(z)=z q(z)$.] Hence $\tau, r, \alpha, V, W$ all depend continuously on $\psi_{0}$.

The restriction of $\Psi$ to $\left\{z:|z| \leqq R \lambda^{2}\right\}$ is also continuous in $\psi_{0}$, since e.g. its Taylor series converges uniformly, and its coefficients depend continuously on $\psi_{0}$.

By the very construction of $\Psi$, the domain of $\Psi$ is the union of an increasing sequence of compacts $\left\{K_{n}\right\}$, with $K_{n-1} \subset \subset K_{n},-\lambda K_{n} \subset \subset K_{n-1}, K_{0}=\left\{z:|z| \leqq \lambda^{2} R\right\}$, such that $\Psi\left(K_{n}\right)$ is contained in the domain of $\hat{V}$. We prove inductively the 
continuous dependence on $\psi_{0}$ of the restriction of $\Psi$ to $K_{n}$, assuming it to hold on $K_{n-1}$. Let $\psi_{1}$ be a function close to $\psi_{0}$ in $-\mathbf{P}\left(\left(-\lambda^{-1}, \lambda^{-2}\right)\right)$, and $\hat{V}_{1}, \Psi_{1}$ the functions obtained from it in the same way as $\hat{V}, \Psi$ from $\psi_{0}$. For a given $\varepsilon>0, \psi_{1}$ can be chosen so close to $\psi_{0}$ that, for all $\zeta \in \Psi\left(K_{n-1}\right)+\Delta(\varepsilon), \hat{V}(\zeta)$ and $\hat{V}_{1}(\zeta)$ are both defined with $\left|\hat{V}(\zeta)-\hat{V}_{1}(\zeta)\right|<\varepsilon / 2, \Delta(\varepsilon)=\left\{z:|z| \leqq \varrho_{\varepsilon}\right\}$. Let $S$ be an upper bound for $\left|\hat{V}^{\prime}\right|$ on $\Psi\left(K_{n-1}\right)+\Delta(\varepsilon)$. Choose $\psi_{1}$ so close to $\psi_{0}$ that, for all $z \in K_{n-1}, \Psi_{1}(z)$ is defined and $\left|\Psi_{1}(z)-\Psi(z)\right|<\min \left(\varrho_{\varepsilon}, \varepsilon / 2 S\right)$. Then for $z \in K_{n}, \Psi_{1}(-\lambda z) \in \Psi\left(K_{n-1}\right)+\Delta(\varepsilon)$, and

$$
\begin{aligned}
\left|\Psi_{1}(z)-\Psi(z)\right| \leqq & \left|\hat{V}\left(\Psi_{1}(-\lambda z)\right)-\hat{V}_{1}\left(\Psi_{1}(-\lambda z)\right)\right| \\
& +\left|\hat{V}\left(\Psi_{1}(-\lambda z)\right)-\hat{V}(\Psi(-\lambda z))\right| \leqq \varepsilon .
\end{aligned}
$$

Thus $\Psi$ depends continuously on $\psi_{0}$. It remains to check that $\gamma$ is also continuous in $\psi_{0}$. This follows from $\gamma=-\psi^{\prime}(0)$, and the fact that the same bounds, obtained for $\psi_{0}^{\prime}(z)$ in (3.5), also hold for $\psi^{\prime}(z)$. In particular, $1-\lambda^{2} \leqq \gamma \leqq 1+\lambda$. Since the domain of analyticity of $\Psi$ around $\gamma$ keeps a finite size, and $\left[\Psi^{\prime}(\gamma)\right]^{-1}$ remains bounded, $\gamma=\Psi^{-1}(1)$ depends continuously on $\psi_{0}$.

The information collected at this point suffices to apply the SchauderTikhonov theorem. Before we do so, we shall devote the two next subsections to obtaining bounds uniform in $\lambda$ in the limit $\lambda \rightarrow 1$.

\subsection{The Functions $H$ and $H_{0}$}

We define:

$$
H(w)=\psi\left(e^{\beta w}\right), \quad H_{0}(w)=\psi_{0}\left(e^{\beta w}\right), \quad \beta=\log \frac{1}{\lambda},
$$

and

$$
\hat{H}=1-H, \quad \hat{H}_{0}=1-H_{0} .
$$

These functions are holomorphic and periodic with period $2 \pi i / \beta$ in $\mathbf{C}$ minus the cuts:

$$
2+\frac{2 m i \pi}{\beta}+\mathbf{R}_{+}, \quad 1+\frac{(2 m+1) i \pi}{\beta}+\mathbf{R}_{+}, \quad m \in \mathbf{Z} .
$$

In particular, $H$ and $H_{0}$ map the strips $\{w: 0< \pm \operatorname{Im} w<\pi / \beta\}$ into $-\mathbf{C}_{ \pm}$, respectively. They tend to 1 when $w$ tends to infinity in the negative real direction. When $w$ is real and increases from $-\infty$ to 2 , they decrease from 1 to $\psi\left(\lambda^{-2}\right)$ and $\psi_{0}\left(\lambda^{-2}\right)$, respectively. They satisfy the functional equations:

$$
H(w)=W(H(w-2)), \quad \hat{H}(w)=\hat{W}(\hat{H}(w-2)),
$$

and

$$
H(0)=H_{0}(0)=0, \quad \hat{H}(0)=\hat{H}_{0}(0)=1
$$

For real $w<2$,

$$
\begin{gathered}
H_{0}^{\prime}(w)=\beta z \psi_{0}^{\prime}(z), \\
\frac{H_{0}^{\prime \prime}(w)}{H_{0}^{\prime}(w)}=\beta\left[1+z \frac{\psi_{0}^{\prime \prime}(z)}{\psi_{0}^{\prime}(z)}\right],
\end{gathered}
$$


where $z=e^{\beta w}$. By (3.6) it follows:

$$
\frac{H_{0}^{\prime \prime}(w)}{H_{0}^{\prime}(w)} \geqq \beta \frac{1-\lambda z}{1+\lambda z},
$$

and this is positive for $z<1 / \lambda$, i.e. $w<1$. Thus $H_{0}$ is concave decreasing on $(-\infty, 1)$. Since $\psi$ obeys the same bounds (3.6) as $\psi_{0}, H$ is also concave decreasing on $(-\infty, 1)$.

We now denote:

$$
w_{1}=-\zeta_{1}=-\log z_{1} / \log \lambda, \quad \text { i.e. } \quad z_{1}=\exp \beta w_{1}, \quad \zeta_{1}=\frac{\log \alpha}{\log (1 / \tau)} .
$$

Then $0<\zeta_{1}<1$, and:

$$
H_{0}\left(w_{1}\right)=\tau, \quad H_{0}^{\prime}\left(w_{1}\right)=-\frac{\tau}{\lambda} \log \frac{1}{\tau} .
$$

Since $H_{0}$ is decreasing concave, and vanishes at 0 ,

$$
\left|H_{0}^{\prime}\left(w_{1}\right)\right|<\frac{H_{0}\left(w_{1}\right)}{\zeta_{1}},
$$

and so:

$$
\log \alpha \leqq \lambda, \quad \alpha \leqq e^{\lambda} .
$$

We now turn to some consequences of the functional equations (3.60). They imply:

$$
\hat{H}(-2 n)=\hat{W}^{-n}(1)
$$

for all $n \in \mathbf{N}$. Let $w \leqq 0$, and denote temporarily $x=\hat{H}(w), y=\hat{H}(w-2)$. Then $0<y<x \leqq 1$, and $x=\hat{W}(y)$, so that, by (3.51),

$$
a y^{3}+y-x \leqq 0 \text {. }
$$

To verify that this implies $y \leqq x\left(1-a^{\prime} x^{2}\right)$, for a certain $a^{\prime}>0$, it suffices to check that, for all $x \in(0,1)$,

$$
a x^{3}\left(1-a^{\prime} x^{2}\right)^{3}-a^{\prime} x^{3} \geqq 0,
$$

i.e.

$$
a\left(1-a^{\prime}\right)^{3}-a^{\prime} \geqq 0 .
$$

Since the left-hand side is $\geqq a\left(1-3 a^{\prime}\right)-a^{\prime}$, this inequality is satisfied by

$$
a^{\prime}=\frac{a}{1+3 a} \text {. }
$$

For $a=1 / 6$, this gives $a^{\prime}=1 / 9$. Thus, for $w \leqq 0$, by the convexity of $\hat{H}$,

$$
\hat{H}^{\prime}(w) \geqq \frac{1}{2}[\hat{H}(w)-\hat{H}(w-2)], \quad \hat{H}^{\prime}(w) \geqq \frac{a^{\prime}}{2} \hat{H}(w)^{3} .
$$


In particular,

$$
\hat{H}^{\prime}(0) \geqq \frac{a^{\prime}}{2}, \quad \text { i.e. } \quad \hat{\psi}^{\prime}(1) \geqq \frac{a^{\prime}}{2 \log (1 / \lambda)} .
$$

Integrating (3.66) with the initial condition $\hat{H}(0)=1$, we find:

$$
\hat{H}(w) \leqq\left(1-a^{\prime} w\right)^{-1 / 2}, \quad w \leqq 0 .
$$

The inequality (3.66) is equivalent to

$$
\beta z \hat{\psi}^{\prime}(z) \geqq \frac{a^{\prime}}{2} \hat{\psi}(z)^{3}, \text { for all } z \in[0,1] .
$$

For any $z \in[0,1], \hat{\psi} \rightarrow\left(\hat{\psi}(z), \beta z \hat{\psi}^{\prime}(z)\right)$ is a continuous linear map of $\mathbf{P}\left(\left(-\lambda^{-1}, \lambda^{-2}\right)\right)$ into $\mathbf{R}^{2}$, and (3.69) requires its image to be contained in the closed convex set $\left\{(X, Y): 2 Y \geqq a^{\prime} X^{3} \geqq 0\right\}$. Therefore:

Lemma 4. The map $M_{\lambda}$ sends into itself the compact convex set

$$
\begin{aligned}
\mathbf{E}_{1}(\lambda)= & \left\{\psi_{0} \in-\mathbf{P}\left(\left(-\lambda^{-1}, \lambda^{-2}\right)\right): \psi_{0}(0)=1, \psi_{0}(1)=0,\right. \\
& \left|\psi_{0}(z)\right| \leqq C_{1}(\lambda) \text { for all } z \in\left(-\lambda^{-1}, \lambda^{-2}\right), \\
& \left.(2 z \log \lambda) \psi_{0}^{\prime}(z) \geqq a^{\prime}\left[1-\psi_{0}(z)\right]^{3} \text { for } z \in[0,1]\right\} .
\end{aligned}
$$

To prove the compactness of $\mathbf{E}_{1}(\lambda)$, note that every function belonging to it maps $\mathbf{C}\left(\left(-\lambda^{-1}, \lambda^{-2}\right)\right)$ into $\mathbf{C}\left(\left(-2 C_{1}(\lambda), 2 C_{1}(\lambda)\right)\right)$, which can be conformally mapped into the unit disk by an obvious transformation, so that $\mathbf{E}_{1}(\lambda)$ is a normal family, and that every limit of functions in $\mathbf{E}_{1}(\lambda)$ is in $\mathbf{E}_{1}(\lambda)$.

From now on, we assume that $\psi_{0}$ is chosen in $\mathbf{E}_{1}(\lambda)$, and that, therefore, the inequalities (3.66)-(3.69) hold with $\hat{H}$ and $\hat{\psi}$ replaced by $\hat{H}_{0}$ and $\hat{\psi}_{0}$ respectively. In particular:

$$
-\psi_{0}^{\prime}(1) \geqq \frac{a^{\prime}}{2 \log 1 / \lambda} .
$$

To conclude this subsection, we note the formula:

$$
V(\zeta)=\frac{1}{\tau} H_{0}\left(\frac{\log (\zeta / \alpha)}{\log (1 / \tau)}\right) .
$$

\subsection{Uniform Lower Bound on $\tau$}

Using the fact that $\psi_{0}$ is now supposed to belong to $\mathbf{E}_{1}(\lambda)$, we can improve the estimates in Subsect. 3.4 so as to get a lower bound on $\tau$ uniform as $\lambda \rightarrow 1$. First by inserting (3.71) into (3.25), we get:

$$
k \leqq \log \frac{2 \log \lambda^{-1}}{\left(1-\lambda^{2}\right) a^{\prime}} \leqq \log \frac{2}{a^{\prime} \lambda(1+\lambda)} .
$$

From this and (3.27), it follows that there is a constant $K_{1}>0$, independent of $\lambda$, such that, for all $\lambda \in(0,1), k \leqq K_{1} \lambda$. Since

$$
\frac{1-\lambda^{2}}{\log 1 / \lambda} \leqq 2,
$$


the constant $K$ of (3.28) can be taken equal to $4 K_{1}+2$, i.e. independent of $\lambda$. Thus $\xi_{1}$ has an upper bound $\xi_{\max }$ which tends to a finite limit as $\lambda \rightarrow 1$, in particular:

$$
\xi_{\max } \leqq 2 K+2 B \log B \text {. }
$$

Second, we note that the bound

$$
\frac{1}{\tau} \leqq \frac{\xi_{\max }-\lambda}{1-\lambda}
$$

is ineffective as $\lambda$ tends to 1 . We therefore use the bound (3.68), as applied to $\hat{H}_{0}$ rather than $\hat{H}$, to get:

$$
\tau=1-\hat{H}_{0}\left(-\zeta_{1}\right) \geqq 1-\left(1+a^{\prime} \zeta_{1}\right)^{-1 / 2},
$$

where $\zeta_{1}$, defined in (3.62), verifies

$$
\zeta_{1} \geqq \frac{1}{\log \lambda} \log \frac{\xi_{\max }-1}{\xi_{\max }-\lambda^{2}} \geqq \frac{\lambda(1+\lambda)}{\xi_{\max }-\lambda^{2}} .
$$

It follows from (3.75) that

$$
\frac{1}{\tau} \leqq \frac{3}{2}+\frac{2}{a^{\prime} \zeta_{1}} \leqq \frac{3}{2}+\frac{2\left(\xi_{\max }-\lambda^{2}\right)}{a^{\prime} \lambda(1+\lambda)}
$$

This bound is well behaved as $\lambda \rightarrow 1$.

\subsection{Existence of Fixed Points}

As a result of the last two subsections, we have:

Lemma 5. There exists a continuous function $\lambda \rightarrow C(\lambda)$ on $(0,1]$ such that, for each $\lambda$ in $(0,1)$, the map $M_{\lambda}$ sends into itself the compact convex set

$$
\begin{aligned}
\mathbf{E}(\lambda)= & \left\{\psi_{0} \in-\mathbf{P}\left(\left(-\lambda^{-1}, \lambda^{-2}\right)\right): \psi_{0}(0)=1, \psi_{0}(1)=0,\right. \\
& \left|\psi_{0}(z)\right| \leqq C(\lambda) \text { for all } z \in\left(-\lambda^{-1}, \lambda^{-2}\right), \\
& \left.(2 z \log \lambda) \psi_{0}^{\prime}(z) \geqq a^{\prime}\left[1-\psi_{0}(z)\right]^{3} \text { for } z \in[0,1]\right\} .
\end{aligned}
$$

Remarks. 1. $\mathbf{E}(\lambda)$ is compact and convex for the same reasons as $\mathbf{E}_{1}(\lambda)$ (which contains it).

2. It has actually been proved that:

$$
\mathbf{E}(\lambda) \subset M_{\lambda}\left(\mathbf{E}_{1}(\lambda)\right), \quad \mathbf{E}_{1}(\lambda) \subset M_{\lambda}\left(\mathbf{E}_{0}(\lambda)\right),
$$

where

$$
\mathbf{E}_{0}(\lambda)=-\mathbf{P}\left(\left(-\lambda^{-1}, \lambda^{-2}\right)\right) \cap\left\{\psi_{0}: \psi_{0}(0)=1, \psi_{0}(1)=0\right\} .
$$

3. It is not difficult, but not very enlightening, to obtain, along the lines suggested at various places in the preceding subsections, an explicit version of $C(\lambda)$.

Applying the Schauder-Tikhonov theorem, we obtain:

Theorem 6. There exists, for each $\lambda \in(0,1)$, at least one fixed point of $M_{\lambda}$ in $\mathbf{E}(\lambda)$. Every fixed point of $M_{\lambda}$ in $\mathbf{E}_{0}(\lambda)$ is in $\mathbf{E}(\lambda)$. 


\subsection{Some Properties of the Fixed Points}

We now assume that a fixed point has been chosen in $\mathbf{E}(\lambda)$ for each $\lambda$ in $(0,1)$, and denote the corresponding functions $\psi_{\lambda}, V_{\lambda}, W_{\lambda}, H_{\lambda}$, etc., keeping the preceding meaning for $\tau, \alpha, r$. These numbers depend, of course, on the choice of the fixed point. Note that:

$$
\begin{aligned}
V_{\lambda}(0) & =\frac{1}{\tau}=\psi_{\lambda}\left(-\lambda^{-1}\right), \quad V_{\lambda}(\alpha)=0, \\
\psi_{\lambda}\left(\lambda^{-2}\right) & =W_{\lambda}(0)=H_{\lambda}(2), \\
V_{\lambda}\left(\alpha \tau^{-2}\right) & =\frac{1}{\tau} W_{\lambda}(0) .
\end{aligned}
$$

We define $y_{0}=1 / \alpha, x_{0}=\alpha^{-1 / r}$, and:

$$
\begin{array}{cc}
U_{\lambda}(z)=y_{0} \psi_{\lambda}(z), & U_{\lambda} \in-\mathbf{P}\left(\left(-\lambda^{-1}, \lambda^{-2}\right)\right), \\
u_{\lambda}(z)=U_{\lambda}(z)^{1 / r}, & u_{\lambda} \in-\mathbf{P}\left(\left(-\lambda^{-1}, 1\right)\right) .
\end{array}
$$

These functions satisfy:

$$
\begin{aligned}
& u_{\lambda}(z)=\frac{1}{\lambda} u_{\lambda}\left(u_{\lambda}(-\lambda z)\right) \quad \text { for all } \quad z \in \mathbf{C}\left(\left(-\lambda^{-1}, 1\right)\right), \\
& U_{\lambda}(1)=u_{\lambda}(1)=0, \quad u_{\lambda}(0)=x_{0}, \quad U_{\lambda}(0)=y_{0} .
\end{aligned}
$$

A straightforward generalization of the results of [9] is possible. We enumerate some salient facts without going into details.

1) The Feigenbaum function restricted to $\left[0, x_{0} / \lambda\right]$ is the inverse function $g_{\lambda}$ of the restriction of $u_{\lambda}$ to $\left[-\lambda^{-1}, 1\right]$. It satisfies all the conditions $\mathbf{C 1}-\mathbf{C 5}$.

2) The function $u_{\lambda}$ has continuous boundary values at the border of $\mathbf{C}\left(\left(-\lambda^{-1}, 1\right)\right)$ and its values there are always non-real except at $-\lambda^{-1}$ and 1 . Its only singularities on the real axis are simple branch points at $(-\lambda)^{-n}$, $n=0,1,2,3, \ldots$. Its continuation across its regularity segments on $\mathbf{R}$ can be studied by the method of [9]. (Note that when $r$ is not an integer, the "domain of analyticity" of $g_{\lambda}$ becomes a ramified Riemann surface.)

3) The functions $\psi_{\lambda}, V_{\lambda}, W_{\lambda}, H_{\lambda}$ are also continuous at the boundaries of the cut planes where they have been defined, and they are bounded there. In fact $u_{\lambda}(-i \infty)$ $=c(\lambda) \in \mathbf{C}_{+}$is a periodic point of period 2 for $u_{\lambda} / \lambda: u_{\lambda}(c(\lambda))=\lambda c(\lambda)^{*}$.

\subsection{Existence of the Eckmann-Wittwer Functions}

Recall (see Subsect. 3.6) that the function $\hat{W}_{\lambda}$ is in $\mathbf{P}((1-\alpha, 1))$ and is bounded in modulus on the real interval $(1-\alpha, 1)$, by $1+1 / \tau \lambda$, with $\alpha>1+\lambda$. On that interval, we have therefore $\left|\hat{W}_{\lambda}(\zeta)\right| \leqq C(\lambda)$. In particular there is a constant $\hat{C}$ such that for all $\lambda \geqq 0.5,\left|\hat{W}_{\lambda}(\zeta)\right|<\hat{C}$ on $(1-\alpha, 1)$. As a result, the functions $\left\{\hat{W}_{\lambda}, \lambda \geqq 0.5\right\}$ form a normal family, and we can find a sequence $\left\{\lambda_{n}\right\}$, tending to 1 , such that the $\hat{W}_{\lambda_{n}}$ converge to $\hat{W}_{1} \in \mathbf{P}((-1,1))$. This function is finite and non-constant, since it must satisfy

$$
\hat{W}_{1}(0)=0, \quad \hat{W}_{1}^{\prime}(0)=1 .
$$


For $0.5 \leqq \lambda<1$, the function $\hat{H}_{\lambda}$ is holomorphic in the domain

$$
\left\{w: 0<|\operatorname{Im} w|<\frac{\pi}{-\log \lambda} \text { or } \operatorname{Im} w=0 \text { and } \operatorname{Re} w<2\right\},
$$

which it maps into $\mathbf{C}_{+} \cup \mathbf{C}_{-} \cup\{|w|<\hat{C}\}$. Hence $\left\{\hat{H}_{\lambda}\right\}$ is also a normal family and, changing to a subsequence if necessary, we can arrange that the $\hat{H}_{\lambda_{n}}$ converge, uniformly on every compact subset of $\mathbf{C}((-\infty, 2))$, to a Herglotz function $\hat{H}_{1}$. The subsequence can also be chosen such that the corresponding $\tau$ and $\alpha$ also have limits $\tau_{1}$ and $\alpha_{1}$. In the limit, we have:

$$
\hat{H}_{1}(w)=\hat{W}_{1}\left(\hat{H}_{1}(w-2)\right), \quad \hat{H}_{1}(0)=1,
$$

and, on the real axis,

$$
0<\hat{H}_{1}(w) \leqq \frac{1}{\sqrt{1-w / 9}}, \quad w \leqq 0 .
$$

Since, for $\lambda<1$, Eq. (3.72) holds, the functions $V_{\lambda_{n}}$ also converge to a function $V_{1} \in-\mathbf{P}\left(\left(0, \alpha_{1} \tau_{1}^{-2}\right)\right)$. This function satisfies $V_{1}(1)=1, V_{1}^{\prime}(1)=-1$, and, at least near $1, V_{1}\left(V_{1}(\zeta)\right)=W_{1}(\zeta) \equiv 1-\hat{W}_{1}(1-\zeta)$. This relation extends analytically in $\mathbf{C}\left(\left(0, \alpha_{1}\right)\right)$.

It is interesting to ask about the fate of the function $\psi_{\lambda_{n}}$ when $n \rightarrow \infty$. Since $\psi_{\lambda}(z)$ $=H_{\lambda}(\log z / \log 1 / \lambda)$, and since $\hat{H}_{\lambda}(w) \leqq(1-w / 9)^{-1 / 2}$ on $(-\infty, 0)$, we see that, if e.g. $z$ is fixed in $(0,1), \psi_{\lambda_{n}}(z) \rightarrow 1$. This is not in contradiction with $\psi_{\lambda}(1)=0$, because 1 is not interior to the limit (intersection in this case) of the domains $\mathbf{C}\left(\left(-\lambda^{-1}, \lambda^{-2}\right)\right)$ as $\lambda$ tends to 1 , but on the boundary of this limit.

\section{The Fixed $r$ Method}

It is tempting to apply the preceding method, with its quasi-tautological estimates, to prove the existence of the Feigenbaum functions for given values of $r$, instead of $\lambda$. This section describes the very limited extent to which this can be carried out, at least in a straightforward way. In this section, $r>1$ is fixed once and for all.

We start again from a function $\psi_{0}=1-\hat{\psi}_{0}$ belonging to $-\mathbf{P}\left(\left(-\lambda_{0}^{-1}, \lambda_{0}^{-2}\right)\right)$, with $\psi_{0}(0)=1$ and $\psi_{0}(1)=0$, where $\lambda_{0} \in(0,1)$ depends on the choice of $\psi_{0}$. We then attempt to define a function $V$ by the same formula (3.7) as in the fixed- $\lambda$ method, the constants $\tau>0$ and $\alpha>1$ being determined by requiring that:

There must exist $\lambda \in(0,1)$ such that:

$$
V(1)=1, \quad V^{\prime}(1)=-\frac{1}{\lambda}, \quad \lambda^{r}=\tau .
$$

This implies that $z_{1}=\alpha^{-1 / r}$ must satisfy

$$
\psi_{0}\left(z_{1}\right)=\tau=\lambda^{r}, \quad \frac{z_{1}}{r} \frac{\psi_{0}^{\prime}\left(z_{1}\right)}{\psi_{0}\left(z_{1}\right)}=-\frac{1}{\lambda},
$$

and hence

$$
-\frac{z_{1}}{r} \psi_{0}^{\prime}\left(z_{1}\right) \psi_{0}\left(z_{1}\right)^{1 / r-1} \equiv-\left.z \frac{d}{d z} \psi_{0}(z)^{1 / r}\right|_{z=z_{1}}=1
$$


There clearly exists a $z_{1} \in(0,1)$ which satisfies $(4.3)$, and it has to be estimated as well as possible. As in Sect. 3,

$$
\frac{1}{1+\lambda_{0} z} \leqq \frac{\psi_{0}(z)}{1-z} \leqq \frac{1}{1-\lambda_{0}^{2} z} \quad\left(0 \leqq z<\lambda_{0}^{-2}\right),
$$

and, for $-\lambda_{0}^{-1}<z<1$,

$$
\frac{\left(1-\lambda_{0}^{2}\right)}{\left(1-\lambda_{0}^{2} z\right)(1-z)} \leqq \frac{-\psi_{0}^{\prime}(z)}{\psi_{0}(z)} \leqq \frac{\left(1+\lambda_{0}\right)}{\left(1+\lambda_{0} z\right)(1-z)} .
$$

The similar bound $\psi_{0}^{\prime \prime}(z) / \psi_{0}^{\prime}(z) \geqq-2 \lambda_{0} /\left(1+\lambda_{0} z\right)$ implies that the left-hand side of (4.3) has a strictly positive derivative in $(0,1)$, so that $(4.3)$ has a unique solution there. Moreover (4.3), (4.4), and (4.5) imply:

$$
\begin{aligned}
& \frac{z_{1}\left(1-\lambda_{0}^{2}\right)}{r\left(1-z_{1}\right)^{1-1 / r}\left(1-\lambda_{0}^{2} z_{1}\right)\left(1+\lambda_{0} z_{1}\right)^{1 / r}} \leqq 1, \\
& 1 \leqq \frac{z_{1}\left(1+\lambda_{0}\right)}{r\left(1-z_{1}\right)^{1-1 / r}\left(1+\lambda_{0} z_{1}\right)\left(1-\lambda_{0}^{2} z_{1}\right)^{1 / r}} .
\end{aligned}
$$

The first and last expressions in (4.6) are increasing in $z_{1}$. For a fixed $\lambda_{0}$, they take the value 0 at $z_{1}=0$, and $+\infty$ at $z_{1}=1$. Hence the first and last inequalities respectively express $z_{1} \leqq z_{\max }\left(\lambda_{0}\right)$ and $z_{1} \geqq z_{\min }\left(\lambda_{0}\right)$. Moreover the first (respectively last) expression in (4.6) is, for a fixed $z_{1}$, decreasing (respectively increasing) in $\lambda_{0}$. Hence $z_{\max }\left(\lambda_{0}\right)$ is an increasing function of $\lambda_{0}$ and $z_{\min }\left(\lambda_{0}\right)$ is a decreasing function of $\lambda_{0}$.

From (4.5) it now follows that

$$
\lambda_{\min }\left(\lambda_{0}\right) \leqq \lambda \leqq \lambda_{\max }\left(\lambda_{0}\right)
$$

where

$$
\begin{aligned}
& \lambda_{\min }\left(\lambda_{0}\right)=\left[\frac{1-z_{\max }\left(\lambda_{0}\right)}{1+\lambda_{0} z_{\max }\left(\lambda_{0}\right)}\right]^{1 / r}, \\
& \lambda_{\max }\left(\lambda_{0}\right)=\left[\frac{1-z_{\min }\left(\lambda_{0}\right)}{1-\lambda_{0}^{2} z_{\min }\left(\lambda_{0}\right)}\right]^{1 / r} .
\end{aligned}
$$

It is easy to verify that [because $\left.z_{\max }^{\prime}\left(\lambda_{0}\right) \geqq 0\right] \lambda_{\min }\left(\lambda_{0}\right)$ is a decreasing function of $\lambda_{0}$, so that $\lambda_{0} \leqq b$ implies $\lambda \geqq \lambda_{\text {min }}\left(\lambda_{0}\right) \geqq \lambda_{\text {min }}(b)$.

To obtain an upper bound on $\lambda$, we can use some of the work expended on the fixed- $\lambda$ method as follows. We note that $z_{1}$ is a zero of the same function $q$ as in (3.10), with the same expression for $A$, viz. $A=A(\lambda)=1 /(-\lambda \log \lambda)$. Since $\log \psi_{0}$ has an integral representation analogous to (3.12), we immediately get:

$$
z_{1} \geqq 1+\lambda \log \lambda \geqq \lambda \text {. }
$$

The difference with the case of Sect. 3 is that the real domain of analyticity of $\psi_{0}$ is now $\left(-\lambda_{0}^{-1}, \lambda_{0}^{-2}\right)$ and that $\sigma$ is known to be equal to 1 on $\left[1, \lambda_{0}^{-2}\right]$, instead of $\left[1, \lambda^{-2}\right]$. 
Assume now that $\lambda \geqq \lambda_{0}$. Then we can repeat all the calculations of Subsect. 3.2, giving lower bounds on $z_{1}$ and $1 / \tau$ : denoting

$$
\xi=\frac{1-\lambda^{2} z}{1-z}, \quad z=\frac{\xi-1}{\xi-\lambda^{2}},
$$

we find again:

$$
\begin{gathered}
\left(1-\lambda^{2}\right) z q(z) \geqq\left(1-\lambda^{2}\right) z q_{2}(z)=\chi(\xi)-\xi, \\
\chi(\xi)=B \log \xi+1+\lambda^{2}-\frac{\lambda^{2}}{\xi} .
\end{gathered}
$$

Recall that $\chi$ is a concave increasing function and that $\chi(\xi)-\xi$ vanishes at 1 and at a unique $\hat{\xi}>1$, which is a lower bound for $\xi_{1}=\left(1-\lambda^{2} z_{1}\right) /\left(1-z_{1}\right)$, itself a lower bound for $1 / \tau$. Since $0<\lambda<1$, we can make the change of variable $\xi=\lambda^{-x}$, with $x>0$. Then if $\hat{\xi}=\lambda^{-\hat{x}}$, it has been seen in Subsect. 3.2 that

$$
\lambda^{2} \leqq \frac{\hat{x}-1}{1+\hat{x}}, \quad \hat{x} \equiv \hat{x}(\lambda)>\frac{1+\lambda^{2}}{1-\lambda^{2}}
$$

Moreover, $0<x<\hat{x}(\lambda)$ is equivalent to $\xi^{-1}[\chi(\xi)-\xi]>0$, which, in terms of $x$, reads:

$$
x \lambda^{x-1}\left(1-\lambda^{2}\right)-\left(1-\lambda^{x}\right)\left(1-\lambda^{x+2}\right) \equiv Z(\lambda, x)>0 .
$$

Since this is a decreasing function of $\xi$ for $\xi \geqq \xi$, it is (for fixed $\lambda$ ) decreasing in $x$ for $x \geqq \hat{x}(\lambda)$. It is easily checked that $\partial Z(\lambda, x) / \partial \lambda>0$ when $\lambda^{2} \leqq(x-1) /(1+x)$. Hence $\hat{x}(\lambda)$ is an increasing function of $\lambda$. The last inequality in (4.14), and an upper bound on $\hat{x}(\lambda)$ which is easy to get (by the same argument as in the remark at the end of Subsect. 3.4) show that $\hat{x}(\lambda) \rightarrow 1$ as $\lambda \rightarrow 0$. We denote $x \rightarrow b(x)$ the function equal to 0 for $0 \leqq x \leqq 1$, and to the inverse function of $\hat{x}$ for $x>1$. The inequality (4.15) is then equivalent to: $\lambda>b(x)$. Since $1 / \tau \geqq \hat{\xi}$, we must have $r \geqq \hat{x}(\lambda)$, and hence

$$
r \lambda^{r-1}\left(1-\lambda^{2}\right)-\left(1-\lambda^{r}\right)\left(1-\lambda^{r+2}\right) \leqq 0
$$

This means that we must have

$$
\lambda \leqq b(r), \quad b(r)^{2}<\frac{r-1}{1+r} .
$$

This proves:

If $b \in[b(r), 1)$, then $\lambda_{0} \leqq b$ implies that $\lambda \leqq b$ and also, as already seen, $\lambda \geqq \lambda_{\min }(b)$.

Choosing $\lambda_{0}$ in $\left[\lambda_{\min }(b(r)), b(r)\right]$, we can take $z_{1}$ as the solution of $(4.3)$ in $(0,1)$ and define $\tau=\psi_{0}\left(z_{1}\right), \lambda=\tau^{1 / r}, \alpha=z_{1}^{-r}<\tau^{-1}$, and $V$ by (3.7). The function $V$ then belongs to $-\mathbf{P}\left(\left(0, \alpha \lambda_{0}^{-2 r}\right)\right)$, vanishes at $\alpha$ and is convex on $(0, \alpha)$ for the same reasons as in Sect. 3. In particular, again, $\alpha \geqq 1+\lambda$. The function $W=V \circ V$ is certainly defined near 1 , and also at $\alpha$, since $W(\alpha)=V(0)=1 / \tau$.

However, in order for $W$ to be defined at 0 , it is necessary that $V(1 / \tau)$ be defined, i.e. that $0<(\alpha \tau)^{-1 / r} \leqq \lambda_{0}^{-2}$, or, equivalently,

$$
z_{1} \lambda_{0}^{2} \leqq \lambda \text {. }
$$


If so, then $W(0)<0$, since $1 / \tau>\alpha$. A sufficient condition for (4.18) to hold is that, for some $b \geqq b(r)$,

$$
1-z_{\max }(b)-\left(1+b z_{\max }(b)\right)\left[b^{2} z_{\max }(b)\right]^{r}>0 .
$$

Let us assume that (4.19) holds. Then $W \in \mathbf{P}((0, \alpha))$. The estimates in Sect. 3, leading to

$$
\hat{W}(\zeta) \equiv 1-W(1-\zeta) \geqq \zeta\left(1+a \zeta^{2}\right),
$$

with $a \geqq 1 / 6$, for $\zeta \in(0,1)$, extend to the present case. Defining, as before,

$$
H_{0}(w)=\psi_{0}\left(\exp \beta_{0} w\right), \quad \beta_{0}=-\log \lambda_{0},
$$

we again find that $H_{0}$ is concave on $(-\infty, 1)$. The same calculations as in Subsect. 3.7 give

$$
W(0) \geqq-\frac{1}{\tau} \frac{z_{1}-\lambda}{\lambda-\lambda_{0}^{2} z_{1}},
$$

and hence

$$
\hat{W}(1) \leqq 1+\frac{1-b^{2}}{\lambda_{\min }^{r}\left(\lambda_{\min }-b^{2} z_{\max }\right)} .
$$

The hypothesis that (4.19) holds implies that this bound is finite for the considered value of $r$. The final steps of the method are the same as in the fixed- $\lambda$ method. To conclude:

Lemma 7. For a fixed value of $r>1$, a sufficient condition for the existence of $a$ fixed point is that there exist a pair $(b, z)$ of numbers in $(0,1)$ satisfying the three following conditions:

$$
\begin{gathered}
r b^{r-1}\left(1-b^{2}\right)-\left(1-b^{r}\right)\left(1-b^{r+2}\right)>0, \\
z\left(1-b^{2}\right)-r(1-z)^{1-1 / r}(1+b z)^{1 / r}\left(1-b^{2} z\right)>0, \\
1-z-(1+b z)\left(b^{2} z\right)^{r}>0 .
\end{gathered}
$$

Recall that (4.22) implies $b>b(r)$, and that $b(r)^{2}<(r-1) /(1+r)$.

For a given $r$, it is easy to verify numerically whether or not the conditions in the lemma can be satisfied, and it appears that the method works for all $r \leqq 14.4$, although we have no proof of the (numerically patent) fact that, if the conditions can be satisfied for a certain $r_{0}$, the same is true for all $r \in\left(1, r_{0}\right)$. The method, however, is particularly easy to apply for small integer values of $r$. For example, in the case $r=2$, it suffices to find $b$ and $z$ in $(0,1)$ such that:

$$
\begin{gathered}
b^{4}+2 b-1>0, \\
z^{2}\left(1-b^{2}\right)^{2}-4(1-z)(1+b z)\left(1-b^{2} z\right)^{2}>0, \\
1-z-(1+b z) z^{2} b^{4}>0 .
\end{gathered}
$$

These conditions are satisfied by $b=1 / 2$ and $z=0.9$. This is obvious for (4.25), and, for (4.27), follows from:

$$
(1-z)=0.1>(1+b) b^{4}=\frac{1.5}{16} .
$$


To verify (4.26), note that

$$
z^{2}\left(1-b^{2}\right)^{2}>0.8 \times \frac{9}{16}=0.45,
$$

and

$$
4(1-z)(1+b z)\left(1-b^{2} z\right)^{2}<0.4 \times \frac{3}{2}(1-0.225)^{2}<0.4 \times \frac{3}{2} \times(0.8)^{2}=0.4 \times 0.96<0.4 \text {. }
$$

The cases $r=3(b=0.65, z=0.9)$ and $r=4(b=0.7, z=0.9)$ are still manageable. Higher values of $r$ require the use of machines. Owners of a pocket calculator can verify, if so inclined, that Table 1 gives, for integer $r$ up to 14, pairs $(b, z)$ satisfying (4.22)-(4.24).

\begin{tabular}{lll} 
Table 1 & & \\
\hline$r$ & $b$ & $z$ \\
\hline 2 & 0.5 & 0.9 \\
3 & 0.65 & 0.9 \\
4 & 0.7 & 0.9 \\
5 & 0.75 & 0.92 \\
6 & 0.78 & 0.93 \\
7 & 0.81 & 0.93 \\
8 & 0.83 & 0.94 \\
9 & 0.85 & 0.94 \\
10 & 0.86 & 0.945 \\
11 & 0.87 & 0.95 \\
12 & 0.88 & 0.95 \\
13 & 0.888 & 0.954 \\
14 & 0.8946 & 0.956 \\
\hline
\end{tabular}

\section{Appendix 1. Proof of Lemma 1}

Denote, for $x \geqq 0$,

$$
f(x)=\left(1-x^{2}\right) \log \left(1+x+x^{2}\right)+2 x^{2} \log x .
$$

To prove that $f(x)>0$ for $0<x<1$, since $f(0)=f(1)=0$, it suffices to prove that $f^{\prime \prime}<0$ on $(0,1)$. Explicit calculations show that $f^{\prime \prime}(x) \rightarrow-\infty$ as $x \rightarrow 0, f^{\prime \prime}(1)$ $=2(1-\log 3)<0$, and $f^{\prime \prime \prime}(x)=P(x) / Q(x)$, where

$$
Q(x)=x\left(1+x+x^{2}\right)^{3}, \quad P(x)=2(1-x)^{2}(1+2 x)(2+x) .
$$

The verification of this is facilitated by noting that $f(x)=-x^{2} f(1 / x)$, hence $f^{\prime \prime \prime}(x)$ $=x^{-4} f^{\prime \prime \prime}(1 / x)$, and, since $Q(1 / x)=x^{-8} Q(x), P(x)=x^{4} P(1 / x)$.

\section{Appendix 2. Direct Proof of Existence for $\lambda=1$}

It is possible to define a fixed point problem directly for $\lambda=1$ (i.e. $r=\infty$ ) by working with the function $H_{0}$ instead of $\psi_{0}$. Let

$$
\begin{aligned}
\mathbf{S}= & \{\hat{H} \in \mathbf{P}((-\infty, 2)): \hat{H}(0)=1,0 \leqq \hat{H}(w) \leqq 10 \text { for } w \in(-\infty, 2), \\
& \left.\hat{H}^{\prime}(w) \geqq \frac{1}{18} \hat{H}(w)^{3} \text { for } w \leqq 0\right\} .
\end{aligned}
$$


The last condition implies:

$$
0<\hat{H}(w) \leqq(1-w / 9)^{-1 / 2} \text { for } w \leqq 0 .
$$

Let $\hat{H}_{0} \in \mathbf{S}$ and $H_{0}=1-\hat{H}_{0}$. Then, in $\mathbf{C}((-\infty, 2))$,

$$
\hat{H}_{0}(w)=\int \frac{d \varrho_{0}(t)}{t-w}, \quad \frac{H_{0}(w)}{w}=-\int \frac{d \varrho_{0}(t)}{t(t-w)},
$$

where $\varrho_{0}$ is a positive measure with support in $[2, \infty)$, such that $\int d \varrho_{0}(t) / t=1$.

The function $V$ will be defined by

$$
V(\zeta)=\frac{1}{\tau} H_{0}\left(\frac{\log (\zeta / \alpha)}{\log (1 / \tau)}\right)
$$

The constants $\tau>0, \alpha>1$ must be such that $V(1)=1=-V^{\prime}(1)$. This implies $Q\left(\zeta_{1}\right)=0$, where

$$
\begin{gathered}
Q(\zeta)=\frac{H_{0}^{\prime}(-\zeta)}{H_{0}(-\zeta)}-\log H_{0}(-\zeta), \\
\zeta_{1}=-w_{1}=-\frac{\log \alpha}{\log \tau}
\end{gathered}
$$

The functions $\log H_{0}$ and $Q$ have integral representations:

$$
\log H_{0}(-\zeta)=-\int_{0}^{\infty} \frac{\sigma(t) d t}{t+\zeta}, \quad Q(\zeta)=\int_{0}^{\infty} \frac{\sigma(t) d t}{t+\zeta}\left[1-\frac{1}{t+\zeta}\right]
$$

(for $\zeta \in \mathbf{C}-\mathbf{R}_{-}$), where $\sigma \in L^{\infty}(\mathbf{R})$ has support in $\mathbf{R}_{+}$, equals 1 on [0,2], and takes values in $[0,1]$ everywhere. It follows from (A.7) that $Q(\zeta)>0$ when $\zeta \geqq 1$. It is clear from (A.5) that $Q(\zeta) \rightarrow-\infty$ when $\zeta \rightarrow 0$. Thus $\zeta_{1}$ exists in $(0,1)$. For more precise bounds, just as in Sect. 3, we split $Q$ as $Q=Q_{1}+Q_{2}$, where $Q_{2}$, the contribution of $[0,2]$ in the integral in (A.7), is given by:

$$
\begin{aligned}
2 Q_{2}(\zeta) & =2 \log \left(1+\frac{2}{\zeta}\right)-\frac{4}{\zeta(\zeta+2)}=\chi(\xi)-\xi, \\
\xi & =1+\frac{2}{\zeta}, \quad \chi(\xi)=2 \log \xi+2-\frac{1}{\xi} .
\end{aligned}
$$

The function $\chi(\xi)-\xi$, just as in Sect. 3, is concave and vanishes at 1 and at a unique $\xi>1$, and $\xi_{1}=1+2 / \zeta_{1}>\xi$. Since $\chi(5)-5>0.01$, it follows that $\xi>5$, and $\zeta_{1}<1 / 2$. Defining $\tau=H_{0}\left(-\zeta_{1}\right)$, and using (A.3), we get:

$$
\frac{1}{\tau} \geqq \xi_{1}>5 \text {. }
$$

The uniqueness of $\zeta_{1}$ follows as in Sect. 3, from:

$$
Q^{\prime}\left(\zeta_{1}\right) \geqq \log \frac{1}{\tau}\left(\log \frac{1}{\tau}-1\right)>\frac{24}{25} .
$$


To obtain a lower bound on $\tau$, we denote

$$
k=\int_{2}^{\infty} \frac{\sigma(t) d t}{t}=-\log \left[-2 H_{0}^{\prime}(0)\right],
$$

and observe that

$$
2 Q(\zeta) \leqq \chi(\xi)-\xi+2 k \equiv S(\xi)
$$

Any $\xi_{\max }>1$ such that $S\left(\xi_{\max }\right)<0$ is an upper bound for $\xi_{1}$, for example $\xi_{\max }=4 \log 4+4 k$. Using (A.1) this gives $\xi_{\max }<15$. From (A.2) it then follows:

$$
\frac{1}{\tau} \leqq \frac{3}{2}+\frac{18}{\zeta_{1}}<128
$$

We now define:

$$
\log \alpha=\zeta_{1} \log \frac{1}{\tau}
$$

so that $1<\alpha<1 / \tau$.

The function $V$ defined by (A.4) is in $-\mathbf{P}\left(\left(0, \alpha \tau^{-2}\right)\right)$. Its iterate $W=V \circ V$ is in $\mathbf{P}((0, \alpha))$, and $W(0)=V(1 / \tau)<0, \quad W(\alpha)=V(0)=1 / \tau$. We also define $\hat{W}(\zeta)$ $=1-W(1-\zeta)$. For $w<2$, it follows from (A.3) that:

$$
\frac{H_{0}^{\prime \prime}(w)}{H_{0}^{\prime}(w)} \leqq \frac{2}{2-w}, \quad \frac{H_{0}^{\prime \prime \prime}(w)}{H_{0}^{\prime}(w)} \leqq \frac{6}{(2-w)^{2}} .
$$

The first of these bounds implies that, for $0<\zeta<\alpha \tau^{-2}$,

$$
-\frac{V^{\prime \prime}(\zeta)}{V^{\prime}(\zeta)} \geqq \frac{1}{\zeta}\left[1-\frac{2}{2 \log (1 / \tau)-\log (\zeta / \alpha)}\right] .
$$

Hence $V$ is convex on $\left(0, \alpha / \tau^{2} e^{2}\right)$, in particular on $(0, \alpha)$, hence $\alpha>2$. Also:

$$
V^{\prime \prime}(1) \geqq 1-\frac{1}{\log (1 / \tau)}>\frac{3}{8} \text {. }
$$

Moreover:

$$
\begin{gathered}
S V(\zeta) \geqq \frac{1}{2 \zeta^{2}}, \quad 0<\zeta<\alpha \tau^{-2} \\
S V(1) \leqq \frac{1}{2}+[\log (1 / \tau)]^{-2} \frac{H_{0}^{\prime \prime \prime}\left(-\zeta_{1}\right)}{H_{0}^{\prime}\left(-\zeta_{1}\right)} \leqq \frac{1}{2}+\frac{3}{2}[\log (1 / \tau)]^{-2} \\
W^{\prime \prime}(1)=0, \quad W^{\prime \prime \prime}(1)=S W(1)=2 S V(1)
\end{gathered}
$$

so that:

$$
\frac{1}{6} \leqq \frac{W^{\prime \prime \prime}(1)}{6} \leqq 0.4
$$

Moreover:

$$
\hat{W}^{(4)}(0)=-W^{(4)}(1)=2 V^{\prime \prime}(1) S V(1)>\frac{3}{8} .
$$


Since $W$ is a Herglotz function, $\hat{W}^{(5)}(\zeta) \geqq 0$ on $(1-\alpha, 1)$, hence $\hat{W}^{(4)}(\zeta)>3 / 8$ on $[0,1)$. Thus

$$
\hat{W}(\zeta) \geqq \zeta+\frac{\zeta^{3}}{6}+\frac{\zeta^{4}}{64} .
$$

To obtain an upper bound on $\hat{W}(1)=1-W(0)$, we note that $W(0)=H_{0}\left(1-\zeta_{1}\right) / \tau$, and integrate the first inequality in (A.15) starting at $-\zeta_{1}$. This gives

$$
\hat{W}(1) \leqq 2 \log \frac{1}{\tau}<10
$$

Definition of $H$ and $\hat{H}$. The next step is to construct a function $\hat{H}=1-H \in \mathbf{S}$, such that, in $\mathbf{C}((-\infty, 2))$,

$$
\hat{H}(w)=\hat{W}(\hat{H}(w-2)), \quad \hat{H}(0)=1 .
$$

To obtain, first, the existence of this function as an element of $\mathbf{P}((-\infty, 2))$, we denote, e.g. for $s \in[0.5,1), \widetilde{W}_{s}=s^{-2} \hat{W}$, and construct a function $\Psi_{s}$ satisfying:

$$
\Psi_{s}(z)=\tilde{W}_{s}\left(\Psi_{s}\left(s^{2} z\right)\right), \quad \Psi_{s}(0)=0, \quad \Psi_{s}^{\prime}(0)=1 .
$$

As in Sect. 3, this function is defined as the limit of the convergent sequence $\tilde{W}_{s}^{n}\left(s^{2 n} z\right)$ in a small disk around 0 , then extended by using (A.26). In $\mathbf{C}_{ \pm}$, this yields a Herglotz function. On the positive real axis $\Psi_{s}$ extends to an increasing function on a certain interval $[0, L)$.

Recall that, on $[0,1), \tilde{W}_{s}$ satisfies $\tilde{W}_{s}(z) \geqq z+a z^{3}$ with $1 / 6 \leqq a<1$. This implies (see Subsect. 3.10) that, on $[0,1], \tilde{W}_{s}^{-1}$ is defined and $\tilde{W}_{s}^{-1}(x) \leqq x-a^{\prime} x^{3}$, with $a^{\prime} \geqq 1 / 9$. Since $\Psi_{s}^{-1}(x)=s^{-2 n} \Psi_{s}^{-1}\left(\tilde{W}_{s}^{-n}(x)\right)$, it is clear that $\Psi_{s}^{-1}(1)=\gamma>0$ exists.

We denote

$$
\tilde{H}_{s}(w)=\Psi_{s}(\gamma \exp (-w \log s)) .
$$

This function is holomorphic in a domain which contains

$$
\Delta_{s}=\left\{w \in \mathbf{C}((-\infty, 2)):|\operatorname{Im} w|<\pi / \log \frac{1}{s}\right\},
$$

and maps $\Delta_{s} \cap \mathbf{C}_{ \pm}$into $\mathbf{C}_{ \pm}$. On $(-\infty, 2), \tilde{H}_{s}$ takes values in $\left(0,10 s^{-2}\right)$. Hence $\left\{\tilde{H}_{s}: 0.5<s<1\right\}$ is a normal family, and the limit of a convergent subsequence (as $s \rightarrow 1)$ yields the required $\hat{H}$. It belongs to $\mathbf{P}((-\infty, 2))$, takes values in $(0,10)$ on $(-\infty, 2)$, satisfies (A.25), and on $(-\infty, 0]$,

$$
\hat{H}(w-2)=\hat{W}^{-1}(\hat{H}(w)) \leqq \hat{H}(w)-\frac{1}{9} \hat{H}(w)^{3},
$$

so that $\hat{H} \in \mathbf{S}$. We define $H=1-\hat{H}$.

Uniqueness and Continuous Dependence of $\hat{H}$ on $\hat{H}_{0}$. It is clear that $\hat{W}$ depends continuously on $\hat{H}_{0}$ in the topology of their respective $\mathbf{F}(J)$. It will now be shown that $\hat{H}$ is unique and depends continuously on $\hat{W}$. Note that

$$
\hat{H}(-2 n)=\hat{W}^{-n}(1), \quad n \in \mathbf{N} \text {. }
$$


Because $\hat{H}$ is in $\mathbf{S}$, it is the unique solution of a very simple and well-known interpolation (or moment) problem. In $\mathbf{C}((-\infty, 2))$,

$$
\hat{H}(w)=\int \frac{d \varrho(t)}{t-w}, \quad \frac{H(w)}{w}=-\int \frac{d \varrho(t)}{t(t-w)}, \quad \int \frac{d \varrho(t)}{t}=1,
$$

where the positive measure $\varrho$ has support in $[2, \infty)$. Let

$$
\Phi(p)=\int_{2}^{\infty} e^{-p t} t^{-1} d \varrho(t) .
$$

This function is holomorphic for $\operatorname{Re} p>0$, continuous and bounded by $\exp (-2 \operatorname{Re} p)$ for $\operatorname{Re} p \geqq 0$. For $\operatorname{Re} \zeta>-2$,

$$
\frac{H(-\zeta)}{\zeta}=\int_{0}^{\infty} e^{-p \zeta} \Phi(p) d p=\int_{-\infty}^{\infty} x^{\frac{\zeta}{2}-1} \mu(x) d x,
$$

where $\mu(x)$ is equal to $\frac{1}{2} \Phi\left(-\frac{1}{2} \log x\right)$ for $x \in[0,1]$ and to 0 elsewhere. Note that $0 \leqq \mu(x) \leqq x / 2$ for all $x \geqq 0$. For any integer $n \geqq 1$,

$$
b_{n} \equiv \frac{H(-2 n)}{2 n}=\int x^{n-1} \mu(x) d x .
$$

The Fourier transform $\tilde{\mu}$ of $\mu$,

$$
\tilde{\mu}(w)=\int e^{i w x} \mu(x) d x
$$

extends to an entire function with modulus bounded by $1 / 2$ on $\mathbf{R}$, and satisfying, for all $w \in \mathbf{C}$,

$$
\left|e^{-i w / 2} \tilde{\mu}(w)\right| \leqq \exp \left|\frac{w}{2}\right|,
$$

and

$$
\tilde{\mu}(w)=\sum_{n=1}^{\infty} \frac{(i w)^{n-1}}{(n-1) !} b_{n} .
$$

It follows that

$$
\left|\tilde{\mu}(w)-\sum_{n=1}^{N} \frac{(i w)^{n-1}}{(n-1) !} b_{n}\right|=\left|\int \mu(x)\left[e^{i w x}-\sum_{n=0}^{N-1} \frac{(i w x)^{n}}{n !}\right] d x\right| \leqq \frac{|w|^{N}}{N !} e^{|w|} .
$$

Let $f$ be a $C^{2}$ function on $\mathbf{R}$, with support in $[0,1]$, such that $\int f(x) x^{k} d x=\delta_{0 k}$, $k=0,1$. Denote

$$
\tilde{f}(w)=\int e^{i w x} f(x) d x,
$$

so that $\tilde{f}(0)=1, \tilde{f}^{\prime}(0)=0$, and

$$
\tilde{v}(w)=\frac{1}{w^{2}}\left[\tilde{\mu}(w)-b_{1} \tilde{f}(w)-i w b_{2} \tilde{f}(w)\right], \quad v(x)=\frac{1}{2 \pi} \int e^{-i w x} \tilde{v}(w) d w,
$$

so that

$$
-v^{\prime \prime}(x)=\mu(x)-b_{1} f(x)+b_{2} f^{\prime}(x) .
$$


Then $\tilde{v}$ is also entire, $v$ has support in $[0,1]$, and there exists a constant $C$, depending only on the choice of $f$, such that:

$$
\left|e^{-i w / 2} \tilde{v}(w)\right| \leqq C \exp \left|\frac{w}{2}\right|, \quad w \in \mathbf{C} ; \quad|\tilde{v}(w)|<\frac{C}{w^{2}+1}, \quad w \in \mathbf{R} .
$$

Given $\varepsilon>0$, one can find $R>0$ such that

$$
\frac{1}{2 \pi} \int_{|w|>R} \frac{C}{w^{2}+1} d w<\frac{\varepsilon}{2}
$$

then $N$ such that

$$
\frac{R^{N-1} e^{R}}{\pi N !}<\frac{\varepsilon}{2}
$$

This ensures that

$$
\left|v(x)-\frac{1}{2 \pi} \int_{-R}^{R} e^{-i w x}\left[\sum_{n=1}^{N} \frac{(i w)^{n-1}}{(n-1) !} b_{n}-b_{1} \tilde{f}(w)-i w b_{2} \hat{f}(w)\right] \frac{d w}{w^{2}}\right|<\varepsilon .
$$

Note that $R$ and $N$ are independent of $\hat{W}$. Finally the formula

$$
\frac{H(-\zeta)}{\zeta}=\int-v(x)\left(\frac{\zeta}{2}-1\right)\left(\frac{\zeta}{2}-2\right) x^{\frac{\zeta}{2}-3} d x+\int\left[b_{1} f(x)-b_{2} f^{\prime}(x)\right] x^{\frac{\zeta}{2}-1} d x
$$

holds when $\operatorname{Re} \zeta>6$, and shows that, in this half-plane, $H$ depends continuously on $H_{0}$. Since $H$ remains in the normal family $\mathbf{S}$, Vitali's theorem shows that $H$ continuously depends on $H_{0}$ as an element of $\mathbf{S}$.

Existence of Fixed Points. The map $T$ defined by $T H_{0}=H$ is continuous on the compact convex set $\mathbf{S}$, which it maps into itself. Hence it has at least one fixed point in $\mathbf{S}$.

Acknowledgements. I am very grateful to Jean-Pierre Eckmann, Oscar Lanford, and Peter Wittwer for their kind encouragement and for very helpful discussions. I also wish to thank the Department of Theoretical Physics of the University of Geneva, whose warm hospitality made some of these discussions possible.

\section{References}

1. Campanino, M., Epstein, H., Ruelle, D.: On Feigenbaum's functional equation. Topology 21, 125-129 (1982). On the existence of Feigenbaum's fixed point. Commun. Math. Phys. 79, 261-302 (1981)

2. Collet, P., Eckmann, J.-P.: Iterated maps of the interval as dynamical systems. Boston: Birkhäuser 1980

3. Collet, P., Eckmann, J.-P., Lanford, O.E. III: Universal properties of maps on the interval. Commun. Math. Phys. 76, 211-254 (1980)

4. Cosnard, M.: Etude des solutions de l'équation fonctionnelle de Feigenbaum. Bifurcations, théorie ergodique et applications. Astérisque 98-99, 143-162 (1982)

5. Coullet, P., Tresser, C.: Itération d'endomorphismes et groupe de renormalisation. J. Phys. Colloq. C 539, C5-25 (1978), C.R. Acad. Sci. Paris 287 A (1978) 
6. Donoghue, W.F. Jr.: Monotone matrix functions and analytic continuation. Berlin, Heidelberg, New York: Springer 1974

7. Dunford, N., Schwartz, J.T.: Linear operators. Part I. General theory. New York: Interscience 1957 (Chap. V.10.5, p. 456)

8. Eckmann, J.-P., Wittwer, P.: Computer methods and Borel summability applied to Feigenbaum's equation. Lecture Notes in Physics, Vol. 227. Berlin, Heidelberg, New York: Springer 1985

9. Epstein, H., Lascoux, J.: Analyticity properties of the Feigenbaum function. Commun. Math. Phys. 81, 437-453 (1981)

10. Falcolini, C.: To appear. (This work extends the method of [1] to cover all values of $r$ in [1,2])

11. Feigenbaum, M.J.: Quantitative universality for a class of non-linear transformations. J. Stat. Phys. 19, 25-52 (1978), Universal metric properties of non-linear transformations. J. Stat. Phys. 21, 669-706 (1979)

12. Lanford, O.E. III: Smooth transformations of intervals. Séminaire N. Bourbaki 1980-1981. Lecture Notes in Mathematics, Vol. 563. Berlin, Heidelberg, New York: Springer 1981

13. Lanford, O.E. III: A computer-assisted proof of the Feigenbaum conjectures. Bull. Am. Math. Soc., New Series 6, 127 (1984)

14. Lanford, O.E. III: A shorter proof of the existence of the Feigenbaum fixed point. Commun. Math. Phys. 96, 521-538 (1984)

15. Valiron, G.: Fonctions analytiques. Paris: Presses Universitaires de France 1954

16. Vul, E.B., Sinai, Ya.G., Khanin, K.M.: Feigenbaum universality and the thermodynamical formalism. Usp. Mat. Nauk 39, 3-37 (1984)

Communicated by O. E. Lanford

Received October 4, 1985 